\title{
A Fourth-Order Compact ADI Scheme for Two-Dimensional Riesz Space Fractional Nonlinear Reaction-Diffusion Equation
}

\author{
Dongdong $\mathrm{Hu}$, Xuenian Cao* \\ School of Mathematics and Computational Science, Xiangtan University, Xiangtan 411105, PR China.
}

\begin{abstract}
In this paper, a second-order backward difference formula (abbr. BDF2) is used to approximate first-order time partial derivative, the Riesz fractional derivatives are approximated by fourth-order compact operators, a class of new alternating-direction implicit difference scheme (abbr. ADI) is constructed for two-dimensional Riesz space fractional nonlinear reaction-diffusion equation. Stability and convergence of the numerical method are analyzed. Numerical experiments demonstrate that the proposed method is effective.
\end{abstract}

Keywords: Riesz space fractional derivative; BDF2 formula; Fourth-order compact operator; ADI scheme; Stability; Convergence

\section{Introduction}

In this paper, we consider two-dimensional Riesz space fractional nonlinear reaction-diffusion equation [19-23, $33,34]$

$$
\frac{\partial u(x, y, t)}{\partial t}=\kappa_{1} \frac{\partial^{\alpha} u(x, y, t)}{\partial|x|^{\alpha}}+\kappa_{2} \frac{\partial^{\beta} u(x, y, t)}{\left.\partial|y|\right|^{\beta}}+g(x, y, t, u(x, y, t)), \quad(x, y, t) \in \Omega \times(0, T],
$$

with the boundary and initial conditions

$$
\begin{gathered}
u(x, y, t)=0, \quad(x, y, t) \in \partial \Omega \times(0, T], \\
u(x, y, 0)=\varphi(x, y), \quad(x, y) \in \bar{\Omega}=\partial \Omega \cup \Omega,
\end{gathered}
$$

where $1<\alpha, \beta<2$ and $\Omega=(a, b) \times(c, d)$, the diffusion coefficients $\kappa_{1}, \kappa_{2}$ are positive constants, $\varphi(x, y)$ is a known sufficiently smooth function, $g(x, y, t, u)$ satisfies the Lipschitz condition

$$
|g(x, y, t, u)-g(x, y, t, v)| \leq L|u-v|, \forall u, v \in \mathbb{R},
$$

here $L$ is Lipschitz constant, and Riesz fractional derivatives $\frac{\partial^{\alpha} u(x, y, t)}{\partial|x|^{\alpha}}$ and $\frac{\partial^{\beta} u(x, y, t)}{\partial|y|^{\beta}}$ are defined by

$$
\frac{\partial^{\alpha} u(x, y, t)}{\partial|x|^{\alpha}}=c_{\alpha}\left({ }_{a}^{R} D_{x}^{\alpha}+{ }_{x}^{R} D_{b}^{\alpha}\right) u(x, y, t), \quad \frac{\partial^{\beta} u(x, y, t)}{\partial|y|^{\beta}}=c_{\beta}\left({ }_{c}^{R} D_{y}^{\beta}+{ }_{y}^{R} D_{d}^{\beta}\right) u(x, y, t),
$$

where $c_{\gamma}=-\frac{1}{2 \cos \left(\frac{\pi y}{2}\right)}, \gamma=\alpha, \beta$, symbols ${ }_{a}^{R} D_{x}^{\alpha},{ }_{x}^{R} D_{b}^{\alpha},{ }_{c}^{R} D_{y}^{\beta}$ and ${ }_{y}^{R} D_{d}^{\beta}$ denote left and right Riemann-Liouville fractional derivative operators, which are defined by [10-13]

$$
{ }_{a}^{R} D_{x}^{\alpha} u(x, y, t)=\frac{1}{\Gamma(2-\alpha)} \frac{\partial^{2}}{\partial x^{2}} \int_{a}^{x} u(\xi, y, t)(x-\xi)^{1-\alpha} d \xi,
$$

\footnotetext{
${ }^{*}$ Corresponding author

Email address: cxn@xtu.edu.cn (Xuenian Cao)
} 


$$
\begin{aligned}
& { }_{x}^{R} D_{b}^{\alpha} u(x, y, t)=\frac{1}{\Gamma(2-\alpha)} \frac{\partial^{2}}{\partial x^{2}} \int_{x}^{b} u(\xi, y, t)(\xi-x)^{1-\alpha} d \xi, \\
& { }_{c}^{R} D_{y}^{\beta} u(x, y, t)=\frac{1}{\Gamma(2-\beta)} \frac{\partial^{2}}{\partial y^{2}} \int_{c}^{y} u(x, \xi, t)(y-\xi)^{1-\beta} d \xi, \\
& { }_{y}^{R} D_{d}^{\beta} u(x, y, t)=\frac{1}{\Gamma(2-\beta)} \frac{\partial^{2}}{\partial y^{2}} \int_{y}^{d} u(x, \xi, t)(\xi-y)^{1-\beta} d \xi,
\end{aligned}
$$

where $\Gamma(\cdot)$ is Gamma function.

In this paper, we assumed that the problem (1.1)-(1.3) has a unique solution $u(x, y, t) \in C_{x, y, t}^{6,6,3}([a, b] \times[c, d] \times[0, T])$. We also supposed for the fixed $t \in[0, T]$ and $y \in[c, d], \widetilde{u}(x, \cdot, \cdot) \in \mathscr{C}^{4+\alpha}(\mathbb{R})$, for the fixed $t \in[0, T]$ and $x \in[a, b]$, $\widetilde{u}(\cdot, y, \cdot) \in \mathscr{C}^{4+\beta}(\mathbb{R})$, where $\widetilde{u}(x, \cdot, \cdot)$ and $\widetilde{u}(\cdot, y, \cdot)$ are defined as

$$
\widetilde{u}(x, \cdot, \cdot)=\left\{\begin{array}{cl}
u(x, \cdot, \cdot), & x \in[a, b], \\
0, & \mathbb{R} \backslash[a, b],
\end{array} \quad \widetilde{u}(\cdot, y, \cdot)=\left\{\begin{array}{cl}
u(\cdot, y, \cdot), & y \in[c, d], \\
0, & \mathbb{R} \backslash[c, d],
\end{array}\right.\right.
$$

here $\mathscr{C}^{4+\alpha}(\mathbb{R})$ and $\mathscr{C}^{4+\beta}(\mathbb{R})$ are of the form

$$
\mathscr{C}^{4+\gamma}(\mathbb{R})=\left\{v\left|v \in L_{1}(\mathbb{R}), \int_{-\infty}^{+\infty}(1+|\varpi|)^{4+\gamma} \sqrt{v}(\varpi)\right| d \varpi<\infty\right\}, \quad \gamma=\alpha, \beta,
$$

where $\widehat{v}(\varpi)$ is represented as the Fourier transformation of $v(x)$ and defined by

$$
\widehat{v}(\varpi)=\int_{-\infty}^{+\infty} e^{-i \varpi x} v(x) d x, \quad i^{2}=-1 .
$$

In recent years, two-dimensional Riesz space fractional nonlinear reaction-diffusion equation plays an essential role in describing the propagation of the electrical potential in heterogeneous cardiac tissue [19-22, 33], it attracts many author's attention in constructing numerical methods for problems of the form (1.1)-(1.3). For approximation of Riesz derivative, Meerschaert and Tadjeran [5] initially proposed the shifted Grünwald-Letnikov approximation with first-order accuracy for Riemann-Liouville fractional derivative. Based on this approximation, Tian et al. [7] estabilished a second-order weighted and shifted Grünwald-Letnikov approximation for Riemann-Liouville fractional derivative, and the approximation was applied in Riesz space fractional advection-dispersion equations [6]. Hao et al. [18] constructed a class of new weighted and shifted Grünwald-Letnikov approximation with second-order accuracy, and it was applied in [8] for fractional Ginzburg-Landau equation. Ortigueira [14] initially proposed the fractional centered difference method with second-order accuracy for Riesz fractional derivative, and this method was applied in Riesz space fractional partial differential equation [3, 15, 16, 21, 24, 25, 32]. Ding and Li [9] proposed a novel second-order approximation for Riesz derivative via constructing a new generating function, and this second-order approximation was adopted in [2] for two-dimension Riesz space-fractional diffusion equation. Recently, compact difference operator has been focused on the fractional differential equations for increasing the spatial accuracy. Zhou et al. [17] constructed a third-order quasi-compact difference scheme for Riemann-Liouville fractional derivative. Hao et al. [18] and Zhao et al. [1] proposed fourth-order compact difference operators to approximate Riemann-Liouville and Riesz derivatives, respectively, these compact difference operators have a great contribution on promoting algorithm accuracy. During these years, there also has developed some approximations by finite element method [22, 28, 29], spectral method [20,33,34] et al.. As we noticed, for the approximation of first-order time derivative, implicit Euler method [19, 28, 30, 31], Crank-Nicolson method [2, 6, 15, 18, 20, 22, 32], implicit midpoint method [8, 26] and BDF2 method [3, 4, 27] are usually used, Padé approximations which are based on Runge-Kutta method are also used in recent researches $[24,25]$. And these methods have their own advantages for time-dependent problems.

There are some researches [19-23, 33, 34] on problem (1.1)-(1.3). Liu et al. [19, 21] constructed two ADI finite difference schemes, where Riesz space derivatives were discretized by shifted Grünwald-Letnikov formulae and fractional centered difference operators, respectively, implicit Euler method was applied to discretize time partial derivative, two proposed methods were proven to be stable and convergent. Bueno-Orovio et al. [33] used Fourier spectral method to approximate Riesz space fractional derivative, implicit Euler method was adopted to discretize firstorder time partial derivative, a semi-implicit Fourier spectral method was developed. Zeng et al. [20] and Bu et al. 
[22] applied Galerkin-Legendre spectral method and Galerkin finite element method to approximate Riesz fractional derivative, respectively, two Crank-Nicolson ADI methods were established. Lin et al. [34] used a bivariate polynomial based on shifted Gegenbauer polynomials method to approximate Riesz space fractional derivative, Runge-Kutta method of order 3 was applied to discretize the first-order time partial derivative, a Runge-Kutta Gegenbauer spectral method was constructed. Iyiola et al. also discussed several implicit-explicit schemes in [23]. Because the computing scale of two dimensional diffusion equation problem is very big, so a more efficient algorithm is needed. So far, there are many high-order algorithms for Riesz fractional derivative, we noticed that the fourth-order fractional compact difference operator in [1] is symmetric positive definite under certain circumstance, it's helpful for us to analyze the stability and convergence. As we know, before ADI method is applied for solving two-dimensional nonlinear reactiondiffusion equation problem. the nonlinear source term needs to have linearized approximation. Therefore, how to deal with the nonlinear source term via linearized approximations [19, 21, 27, 35] plays an important role in constructing ADI scheme for two-dimensional Riesz space fractional nonlinear reaction-diffusion equation. The objective of this paper is to try to use BDF method and the fourth-order fractional compact difference operator to construct a class of new high accuracy ADI scheme based on the first-order [19] and second-order [35] linearized approximations for nonlinear source term. Stability and convergence analysis are given by energy method.

The outline of this paper is organized as follows. In Section 2, the numerical method is constructed for problem (1.1)-(1.3). Then in Section 3, stability and convergence are discussed, respectively. In Section 4, we use the proposed method and these methods in literatures $[19,21]$ to solve the test problems. Numerical results show that the proposed method has high accuracy and efficiency.

\section{Numerical method}

Let $x_{i}=a+i h_{x}, i=0,1,2, \cdots, M_{1}, y_{j}=c+j h_{y}, j=0,1,2, \cdots, M_{2}, t_{n}=n \tau, n=0,1,2, \cdots, N$, where $h_{x}=(b-a) / M_{1}$ and $h_{y}=(d-c) / M_{2}$ are spatial step sizes, $\tau=T / N$ denotes time step size. $u\left(x_{i}, y_{j}, t_{n}\right)$ and $u_{i, j}^{n}$ are exact solution and numerical solution of the problem (1.1)-(1.3) at $\left(x_{i}, y_{j}, t_{n}\right)$, respectively. We also denote $\bar{\Omega}_{h}=\left\{\left(x_{i}, y_{j}\right) \mid 0 \leq i \leq M_{1}, 0 \leq j \leq M_{2}\right\}, \Omega_{h}=\bar{\Omega}_{h} \cap \Omega$, and the boundary grid mesh is $\partial \Omega_{h}=\bar{\Omega}_{h} \cap \partial \Omega$.

To discretize the Riesz space fractional derivative, we would introduce the centred difference operators which are defined by [14]

$$
\Delta_{x}^{\alpha} u\left(x_{i}, y_{j}, t_{n}\right)=\frac{-1}{h_{x}^{\alpha}} \sum_{k=i-M_{1}}^{i} g_{k}^{(\alpha)} u\left(x_{i}-k h_{x}, y_{j}, t_{n}\right)
$$

and

$$
\Delta_{y}^{\beta} u\left(x_{i}, y_{j}, t_{n}\right)=\frac{-1}{h_{y}^{\beta}} \sum_{k=j-M_{2}}^{j} g_{k}^{(\beta)} u\left(x_{i}, y_{j}-k h_{y}, t_{n}\right),
$$

where the coefficients $g_{k}^{(\gamma)}$ are determined by

$$
g_{0}^{(\gamma)}=\frac{\Gamma(\alpha+1)}{\Gamma^{2}(\alpha / 2+1)}, \quad g_{k}^{(\gamma)}=\left(1-\frac{\alpha+1}{\alpha / 2+k}\right) g_{k-1}^{(\gamma)}, \quad g_{-k}^{(\gamma)}=g_{k}^{(\gamma)}, \quad \gamma=\alpha, \beta, \quad k=1,2, \cdots,
$$

then we have following lemma.

Lemma 2.1. (see [1].) If $\widetilde{u}(x, \cdot, \cdot) \in \mathscr{C}^{4+\alpha}(\mathbb{R}), \widetilde{u}(\cdot, y, \cdot) \in \mathscr{C}^{4+\beta}(\mathbb{R})$, for the fixied step-sizes $h_{x}$ and $h_{y}$, it holds that

$$
\begin{array}{ll}
\mathscr{B}_{x}^{\alpha} \frac{\partial^{\alpha} u\left(x_{i}, y_{j}, t_{n}\right)}{\partial|x|^{\alpha}}=\Delta_{x}^{\alpha} u\left(x_{i}, y_{j}, t_{n}\right)+O\left(h_{x}^{4}\right), & 1 \leq i \leq M_{1}-1,1 \leq j \leq M_{2}-1,0 \leq n \leq N, \\
\mathscr{B}_{y}^{\beta} \frac{\partial^{\beta} u\left(x_{i}, y_{j}, t_{n}\right)}{\partial|y|^{\beta}}=\Delta_{y}^{\beta} u\left(x_{i}, y_{j}, t_{n}\right)+O\left(h_{y}^{4}\right), & 1 \leq i \leq M_{1}-1,1 \leq j \leq M_{2}-1,0 \leq n \leq N,
\end{array}
$$

where the Fourth-order compact operators $\mathscr{B}_{x}^{\alpha}$ and $\mathscr{B}_{y}^{\beta}$ are defined as follows

$$
\mathscr{B}_{x}^{\alpha} u\left(x_{i}, y_{j}, t_{n}\right)= \begin{cases}c_{2}^{\alpha} u\left(x_{i-1}, y_{j}, t_{n}\right)+\left(1-2 c_{2}^{\alpha}\right) u\left(x_{i}, y_{j}, t_{n}\right)+c_{2}^{\alpha} u\left(x_{i+1}, y_{j}, t_{n}\right), & 1 \leq i \leq M_{1}-1,0 \leq j \leq M_{2}, \\ u\left(x_{i}, y_{j}, t_{n}\right), & i=\left\{0, M_{1}\right\}, 0 \leq j \leq M_{2} .\end{cases}
$$


and

$$
\mathscr{B}_{y}^{\beta} u\left(x_{i}, y_{j}, t_{n}\right)= \begin{cases}c_{2}^{\beta} u\left(x_{i}, y_{j-1}, t_{n}\right)+\left(1-2 c_{2}^{\beta}\right) u\left(x_{i}, y_{j}, t_{n}\right)+c_{2}^{\beta} u\left(x_{i}, y_{j+1}, t_{n}\right), & 1 \leq j \leq M_{2}-1,0 \leq i \leq M_{1}, \\ u\left(x_{i}, y_{j}, t_{n}\right), & j=\left\{0, M_{2}\right\}, 0 \leq i \leq M_{1},\end{cases}
$$

where $c_{2}^{\gamma}=\frac{\gamma}{24} \in\left(\frac{1}{24}, \frac{1}{12}\right), \gamma=\alpha, \beta$.

Before approximating the first-order partial derivative, we would introduce the properties of BDF operator.

Lemma 2.2. (see [3].) For any positive integer $n$, if $u(\cdot, \cdot, t) \in C^{3}([0, T])$, then

$$
\frac{\partial u\left(x_{i}, y_{j}, t_{n}\right)}{\partial t}=D_{t}^{(2)} u\left(x_{i}, y_{j}, t_{n}\right)+r_{i, j}^{n}
$$

where

$$
\begin{gathered}
D_{t}^{(2)} u\left(x_{i}, y_{j}, t_{n}\right)= \begin{cases}\delta_{t} u\left(x_{i}, y_{j}, t_{\frac{1}{2}}\right), & n=1, \\
\frac{3}{2} \delta_{t} u\left(x_{i}, y_{j}, t_{n-\frac{1}{2}}\right)-\frac{1}{2} \delta_{t} u\left(x_{i}, y_{j}, t_{n-\frac{3}{2}}\right), & n \geq 2,\end{cases} \\
\delta_{t} u\left(x_{i}, y_{j}, t_{n-\frac{1}{2}}\right)=\frac{1}{\tau}\left(u\left(x_{i}, y_{j}, t_{n}\right)-u\left(x_{i}, y_{j}, t_{n-1}\right)\right),
\end{gathered}
$$

and $r_{i, j}^{n}$ satisfies

$$
\left|r_{i, j}^{n}\right|= \begin{cases}O(\tau), & n=1 \\ O\left(\tau^{2}\right), & n \geq 2\end{cases}
$$

The nonlinear source term can be treated by following process

$$
g\left(x_{i}, y_{j}, t_{n}, u\left(x_{i}, y_{j}, t_{n}\right)\right)= \begin{cases}g\left(x_{i}, y_{j}, t_{1}, u\left(x_{i}, y_{j}, t_{0}\right)\right)+O(\tau), & n=1, \\ g\left(x_{i}, y_{j}, t_{n}, 2 u\left(x_{i}, y_{j}, t_{n-1}\right)-u\left(x_{i}, y_{j}, t_{n-2}\right)\right)+O\left(\tau^{2}\right), & n \geq 2\end{cases}
$$

Let

$$
\widehat{g}\left(x_{i}, y_{j}, t_{n}, u\left(x_{i}, y_{j}, t_{n}\right)\right)= \begin{cases}g\left(x_{i}, y_{j}, t_{1}, u\left(x_{i}, y_{j}, t_{0}\right)\right), & n=1 \\ g\left(x_{i}, y_{j}, t_{n}, 2 u\left(x_{i}, y_{j}, t_{n-1}\right)-u\left(x_{i}, y_{j}, t_{n-2}\right)\right), & n \geq 2 .\end{cases}
$$

At the point $\left(x_{i}, y_{j}, t_{n}\right)(1.1)$ becomes

$$
\frac{\partial u\left(x_{i}, y_{j}, t_{n}\right)}{\partial t}=\kappa_{1} \frac{\partial^{\alpha} u\left(x_{i}, y_{j}, t_{n}\right)}{\partial|x|^{\alpha}}+\kappa_{2} \frac{\partial^{\beta} u\left(x_{i}, y_{j}, t_{n}\right)}{\partial|y|^{\beta}}+g\left(x_{i}, y_{j}, t_{n}, u\left(x_{i}, y_{j}, t_{n}\right)\right), \quad\left(x_{i}, y_{j}\right) \in \Omega_{h}, 1 \leq n \leq N .
$$

Multiplying by $\mathscr{B}_{x}^{\alpha} \mathscr{B}_{y}^{\beta}$ in (2.8), we obtain from Lemma 2.1 that

$$
\mathscr{B}_{x}^{\alpha} \mathscr{B}_{y}^{\beta} \frac{\partial u\left(x_{i}, y_{j}, t_{n}\right)}{\partial t}=\mathscr{B}_{y}^{\beta} \delta_{x}^{\alpha} u\left(x_{i}, y_{j}, t_{n}\right)+\mathscr{B}_{x}^{\alpha} \delta_{y}^{\beta} u\left(x_{i}, y_{j}, t_{n}\right)+\mathscr{B}_{x}^{\alpha} \mathscr{B}_{y}^{\beta} g\left(x_{i}, y_{j}, t_{n}, u\left(x_{i}, y_{j}, t_{n}\right)\right)+O\left(h_{x}^{4}+h_{y}^{4}\right),
$$

where $\delta_{x}^{\alpha}=\kappa_{1} \Delta_{x}^{\alpha}$ and $\delta_{y}^{\alpha}=\kappa_{2} \Delta_{y}^{\alpha}$.

Substituting (2.6) into (2.9), we obtain

$$
\begin{aligned}
\mathscr{B}_{x}^{\alpha} \mathscr{B}_{y}^{\beta} D_{t}^{(2)} u\left(x_{i}, y_{j}, t_{n}\right)= & \mathscr{B}_{y}^{\beta} \delta_{x}^{\alpha} u\left(x_{i}, y_{j}, t_{n}\right)+\mathscr{B}_{x}^{\alpha} \delta_{y}^{\beta} u\left(x_{i}, y_{j}, t_{n}\right) \\
& +\mathscr{B}_{x}^{\alpha} \mathscr{B}_{y}^{\beta} g\left(x_{i}, y_{j}, t_{n}, u\left(x_{i}, y_{j}, t_{n}\right)\right)-\mathscr{B}_{x}^{\alpha} \mathscr{B}_{y}^{\beta} r_{i, j}^{n}+O\left(h_{x}^{4}+h_{y}^{4}\right) .
\end{aligned}
$$

Adding a small error term $\tau^{2} \sigma_{n}^{2} \delta_{x}^{\alpha} \delta_{y}^{\beta} D_{t}^{(2)} u\left(x_{i}, y_{j}, t_{n}\right)$ on both side of (2.10), we have

$$
\begin{aligned}
\mathscr{B}_{x}^{\alpha} \mathscr{B}_{y}^{\beta} D_{t}^{(2)} u\left(x_{i}, y_{j}, t_{n}\right)+\tau^{2} \sigma_{n}^{2} \delta_{x}^{\alpha} \delta_{y}^{\beta} D_{t}^{(2)} u\left(x_{i}, y_{j}, t_{n}\right)= & \mathscr{B}_{y}^{\beta} \delta_{x}^{\alpha} u\left(x_{i}, y_{j}, t_{n}\right)+\mathscr{B}_{x}^{\alpha} \delta_{y}^{\beta} u\left(x_{i}, y_{j}, t_{n}\right) \\
& +\mathscr{B}_{x}^{\alpha} \mathscr{B}_{y}^{\beta} \widehat{g}\left(x_{i}, y_{j}, t_{n}, u\left(x_{i}, y_{j}, t_{n}\right)\right)+\mathscr{R}_{i, j}^{n},
\end{aligned}
$$

where $\sigma_{1}=1$ and $\sigma_{n}=\frac{2}{3}, n \geq 2$. And there exists the positive constants $c_{1}$ and $c_{2}$ such that

$$
\left|\mathscr{R}_{i, j}^{n}\right| \leq \begin{cases}c_{1}\left(\tau+h_{x}^{4}+h_{y}^{4}\right), & n=1 \\ c_{2}\left(\tau^{2}+h_{x}^{4}+h_{y}^{4}\right), & n \geq 2\end{cases}
$$


Omitting the truncation error $\mathscr{R}_{i, j}^{n}$, we can obtain the numerical scheme for solving the problem (1.1)-(1.3) as follows

$$
\mathscr{B}_{x}^{\alpha} \mathscr{B}_{y}^{\beta} D_{t}^{(2)} u_{i, j}^{n}+\tau^{2} \sigma_{n}^{2} \delta_{x}^{\alpha} \delta_{y}^{\beta} D_{t}^{(2)} u_{i, j}^{n}=\mathscr{B}_{y}^{\beta} \delta_{x}^{\alpha} u_{i, j}^{n}+\mathscr{B}_{x}^{\alpha} \delta_{y}^{\beta} u_{i, j}^{n}+\mathscr{B}_{x}^{\alpha} \mathscr{B}_{y}^{\beta} g_{i, j}^{n}, \quad\left(x_{i}, y_{j}\right) \in \Omega_{h}, 1 \leq n \leq N
$$

where

$$
g_{i, j}^{n}= \begin{cases}g\left(x_{i}, y_{j}, t_{1}, u_{i, j}^{0}\right), & n=1, \\ g\left(x_{i}, y_{j}, t_{n}, 2 u_{i, j}^{n-1}-u_{i, j}^{n-2}\right), & n \geq 2,\end{cases}
$$

the boundary and initial conditions are

$$
\begin{gathered}
u_{i, j}^{n}=0, \quad\left(x_{i}, y_{j}\right) \in \partial \Omega_{h}, 1 \leq n \leq N, \\
u_{i, j}^{0}=\varphi\left(x_{i}, y_{j}\right), \quad\left(x_{i}, y_{j}\right) \in \bar{\Omega}_{h} .
\end{gathered}
$$

Multipling by $\tau \sigma_{n}$ in (2.13), and factorizing it, we have

$$
\left(\mathscr{B}_{x}^{\alpha}-\tau \sigma_{n} \delta_{x}^{\alpha}\right)\left(\mathscr{B}_{y}^{\beta}-\tau \sigma_{n} \delta_{y}^{\beta}\right) u_{i, j}^{n}=\mathscr{H} u_{i, j}^{n-1}+\tau \sigma_{n} \mathscr{B}_{x}^{\alpha} \mathscr{B}_{y}^{\beta} g_{i, j}^{n},
$$

where $\mathscr{H} u_{i, j}^{n-1}=\left(\mathscr{B}_{x}^{\alpha} \mathscr{B}_{y}^{\beta}+\tau^{2} \sigma_{n}^{2} \delta_{x}^{\alpha} \delta_{y}^{\beta}\right)\left(I-\sigma_{n} \tau D_{t}^{(2)}\right) u_{i, j}^{n}$, and we can also rewrite it as follows

$$
\mathscr{H} u_{i, j}^{n-1}= \begin{cases}\left(\mathscr{B}_{x}^{\alpha} \mathscr{B}_{y}^{\beta}+\tau^{2} \delta_{x}^{\alpha} \delta_{y}^{\beta}\right) u_{i, j}^{0}, & n=1, \\ \left(\mathscr{B}_{x}^{\alpha} \mathscr{B}_{y}^{\beta}+\frac{4}{9} \tau^{2} \delta_{x}^{\alpha} \delta_{y}^{\beta}\right)\left(\frac{4}{3} u_{i, j}^{n-1}-\frac{1}{3} u_{i, j}^{n-2}\right), & n \geq 2 .\end{cases}
$$

Introducing an intermediate variable $u_{i, j}^{*}$, let $u_{i, j}^{*}=\left(\mathscr{B}_{y}^{\beta}-\tau \sigma_{n} \delta_{y}^{\beta}\right) u_{i, j}^{n}$, therefore, we constructed a class of D'Yakonov ADI finite difference scheme for solving the problem (1.1)-(1.3) as follows

Step 1: for the fixed $j \in\left\{1,2, \cdots, M_{2}-1\right\},\left\{u_{i, j}^{*} \mid 1 \leq i \leq M_{1}-1\right\}$ can be calculated by

$$
\left(\mathscr{B}_{x}^{\alpha}-\tau \sigma_{n} \delta_{x}^{\alpha}\right) u_{i, j}^{*}=\mathscr{H} u_{i, j}^{n-1}+\tau \sigma_{n} \mathscr{B}_{x}^{\alpha} \mathscr{B}_{y}^{\beta} g_{i, j}^{n}, \quad 1 \leq n \leq N,
$$

with the boundary conditions

$$
u_{0, j}^{*}=\left(\mathscr{B}_{y}^{\beta}-\tau \sigma_{n} \delta_{y}^{\beta}\right) u_{0, j}^{n}, \quad u_{M_{1}, j}^{*}=\left(\mathscr{B}_{y}^{\beta}-\tau \sigma_{n} \delta_{y}^{\beta}\right) u_{M_{1}, j}^{n}, \quad 1 \leq j \leq M_{2}-1, \quad 1 \leq n \leq N .
$$

Step 2: for the fixed $i \in\left\{1,2, \cdots, M_{1}-1\right\},\left\{u_{i, j}^{n} \mid 1 \leq j \leq M_{2}-1\right\}$ can be obtained by

$$
\left(\mathscr{B}_{y}^{\beta}-\tau \sigma_{n} \delta_{y}^{\beta}\right) u_{i, j}^{n}=u_{i, j}^{*}, \quad 1 \leq n \leq N,
$$

the boundary and initial conditions are (2.14)-(2.15).

\section{Stability and convergence analysis}

In order to analyze the stability and convergence of the method, we introduce some notations and lemmas.

Let

$$
\widehat{\gamma}_{h}=\left\{\zeta^{n} \mid \zeta^{n}=\left(\zeta_{0,0}^{n}, \cdots, \zeta_{M_{1}, 0}^{n}, \cdots, \zeta_{0, M_{2}}^{n}, \cdots, \zeta_{M_{1}, M_{2}}^{n}\right), \zeta_{i, j}^{n}=0 \text { if }\left(x_{i}, y_{j}\right) \in \partial \Omega_{h}, 0 \leq n \leq N\right\} .
$$

For any $u^{n}, v^{n} \in \widehat{\gamma}_{h}$, we define the following discrete inner product and corresponding norm

$$
\left(u^{n}, v^{n}\right)=h_{x} h_{y} \sum_{i=1}^{M_{1}-1} \sum_{j=1}^{M_{2}-1} u_{i, j}^{n} v_{i, j}^{n}, \quad\left\|u^{n}\right\|=\sqrt{\left(u^{n}, u^{n}\right)} .
$$

In addition, for any $u^{n} \in \widehat{\gamma}_{h}$, we denote $\left|u^{n}\right|=\left(\left|u_{0,0}^{n}\right|, \cdots,\left|u_{M_{1}, 0}^{n}\right|, \cdots,\left|u_{0, M_{2}}^{n}\right|, \cdots,\left|u_{M_{1}, M_{2}}^{n}\right|\right)$, it implies $\left|u^{n}\right| \in \widehat{\gamma}_{h}$, and denote $u_{*, j}^{n}=\left(u_{0, j}^{n}, \cdots, u_{M_{1}, j}^{n}\right)$ and $u_{i, *}^{n}=\left(u_{i, 0}^{n}, \cdots, u_{i, M_{2}}^{n}\right)$. 
Lemma 3.1. (see [3].) For any positive integer $n$ and real vector $v=\left(v^{0}, v^{1}, \cdots, v^{n}\right) \in \mathbb{R}^{n+1}$, we have

$$
\begin{array}{ll}
\frac{4 \tau}{3} \sum_{k=2}^{n} v^{k}\left(D_{t}^{(2)} v^{k}\right) \geq\left(v^{n}\right)^{2}-\frac{1}{3}\left(v^{n-1}\right)^{2}-\left(v^{1}\right)^{2}+\frac{1}{3}\left(v^{0}\right)^{2}-\frac{2}{3}\left(v^{1}-v^{0}\right)^{2}, & n \geq 2, \\
\frac{4 \tau}{3} \sum_{k=1}^{n} v^{k}\left(D_{t}^{(2)} v^{k}\right) \geq\left(v^{n}\right)^{2}-\frac{1}{3}\left(v^{n-1}\right)^{2}-\frac{1}{3}\left(v^{1}\right)^{2}-\frac{1}{3}\left(v^{0}\right)^{2}, & n \geq 1, \\
\frac{4 \tau}{3} v^{1}\left(D_{t}^{(2)} v^{1}\right)=\frac{2}{3}\left(v^{1}\right)^{2}-\frac{2}{3}\left(v^{0}\right)^{2}+\frac{2}{3}\left(v^{1}-v^{0}\right)^{2}, & n=1 .
\end{array}
$$

It is easy to check that $\mathscr{B}_{x}^{\alpha}$ and $\mathscr{B}_{y}^{\beta}$ are symmetric positive definite and self-adjoint [1], Following from Lemma 3.11 in [1], one can prove that there exists the fractional symmetric positive definite difference operators $Q_{x}$ and $Q_{y}$ such that $\mathscr{B}_{x}^{\alpha}=\left(Q_{x}\right)^{2}$ and $\mathscr{B}_{y}^{\beta}=\left(Q_{y}\right)^{2}$, here, $Q_{x}$ and $Q_{y}$ are also commutable.

Lemma 3.2. (see [1].) For any $u^{n} \in \widehat{\gamma}_{h}$, it holds that

$$
\frac{1}{3}\left\|u^{n}\right\|^{2} \leq\left\|u^{n}\right\|_{\mathscr{B}}^{2} \leq\left\|u^{n}\right\|^{2},
$$

where $\left\|u^{n}\right\|_{\mathscr{B}}=\sqrt{\left(\mathscr{B}_{x}^{\alpha} \mathscr{B}_{y}^{\beta} u^{n}, u^{n}\right)}=\sqrt{\left(Q_{x} Q_{y} u^{n}, Q_{x} Q_{y} u^{n}\right)}$.

Lemma 3.3. (see $[1,3,15]$.) For any $u^{n} \in \widehat{\gamma}_{h}$, it holds that

$$
\begin{array}{ll}
\left(\delta_{x}^{\alpha} u_{*, j}^{n}, u_{*, j}^{n}\right):=-\left(\Lambda_{x} u_{*, j}^{n}, \Lambda_{x} u_{*, j}^{n}\right) \leq 0, & 1 \leq j \leq M_{2}-1, \\
\left(\delta_{y}^{\beta} u_{i, *}^{n}, u_{i, *}^{n}\right):=-\left(\Lambda_{y} u_{i, *}^{n}, \Lambda_{y} u_{i, *}^{n}\right) \leq 0, & 1 \leq i \leq M_{1}-1,
\end{array}
$$

where $\Lambda_{x}, \Lambda_{y}$ are represented as fractional symmetric positive definite difference operators such that $-\delta_{x}^{\alpha}=\left(\Lambda_{x}\right)^{2}$, $-\delta_{y}^{\beta}=\left(\Lambda_{y}\right)^{2}$.

It is easy to verify that

$$
\left(\delta_{x}^{\alpha} \delta_{y}^{\beta} u^{n}, u^{n}\right) \geq 0 .
$$

Theorem 4.1 in [15] means that $-\delta_{x}^{\alpha}$ and $-\delta_{y}^{\beta}$ are symmetric positive definite operators. Therefore, with the help of commutativity of $\Lambda_{x}$ and $\Lambda_{y}$, we could introduce a semi-norm $\left\|u^{n}\right\|_{\delta}^{2} \triangleq\left(\delta_{x}^{\alpha} \delta_{y}^{\beta} u^{n}, u^{n}\right):=\left(\Lambda_{x} \Lambda_{y} u^{n}, \Lambda_{x} \Lambda_{y} u^{n}\right)$ be similar with $[1,3]$.

Lemma 3.4. For any $u^{n} \in \widehat{\gamma}_{h}$, it holds that

$$
\left(\mathscr{B}_{y}^{\beta} \delta_{x}^{\alpha} u^{n}, u^{n}\right) \leq 0, \quad\left(\mathscr{B}_{x}^{\alpha} \delta_{y}^{\beta} u^{n}, u^{n}\right) \leq 0 .
$$

Proof. From Lemma 3.3, we have

$$
\begin{aligned}
\left(\mathscr{B}_{y}^{\beta} \delta_{x}^{\alpha} u^{n}, u^{n}\right) & =\left(\delta_{x}^{\alpha} Q_{y} u^{n}, Q_{y} u^{n}\right)=h_{x} h_{y} \sum_{i=1}^{M_{1}-1} \sum_{j=1}^{M_{2}-1}\left(\delta_{x}^{\alpha} Q_{y} u_{i, j}^{n}\right)\left(Q_{y} u_{i, j}^{n}\right) \\
& =h_{y} \sum_{j=1}^{M_{2}-1}\left(\delta_{x}^{\alpha} Q_{y} u_{*, j}^{n}, Q_{y} u_{*, j}^{n}\right) \\
& \leq 0 .
\end{aligned}
$$

Similarly, we can obtain

$$
\left(\mathscr{B}_{x}^{\alpha} \delta_{y}^{\beta} u^{n}, u^{n}\right) \leq 0
$$

The proof is completed.

Lemma 3.5. (Discrete Bellman Inequality) Let $\rho_{1}, \rho_{2} \geqslant 0, \tau>0, \epsilon_{0}, \epsilon_{1}, \cdots, \epsilon_{\widehat{N}}$ are a series of nonnegative real numbers, satisfying

$$
\epsilon_{n} \leq \rho_{2}+\rho_{1} \tau \sum_{k=0}^{n-1} \epsilon_{k}, \quad n=1, \cdots, \widehat{N},
$$

then it holds that

$$
\epsilon_{n} \leq \rho_{2} e^{\rho_{1} n \tau}, \quad n=1, \cdots, \widehat{N} .
$$


Assuming that $\widetilde{u}_{i, j}^{n}$ is the numerical solution for the numerical method (2.13)-(2.15) starting from another initial value. Denote $E^{n}=\left(E_{0,0}^{n}, \cdots, E_{M_{1}, 0}^{n}, \cdots, E_{0, M_{2}}^{n}, \cdots, E_{M_{1}, M_{2}}^{n}\right)$, where $E_{i, j}^{n}=u_{i, j}^{n}-\widetilde{u}_{i, j}^{n},\left(x_{i}, y_{j}\right) \in \Omega_{h}$, then we have following consequence.

Theorem 3.1. For any positive real number $v \in(0,1)$, if $0<\tau \leq \tau_{0}=\frac{1-v}{9 L}$, then the numerical scheme (2.13)-(2.15) is stable, i.e.

$$
\left\|E^{n}\right\| \leq \frac{3}{v} e^{\frac{18}{v} L T}\left\|\left(I+\frac{23}{18} \tau^{2} \delta_{x}^{\alpha} \delta_{y}^{\beta}\right) E^{0}\right\|, \quad n \geq 1
$$

Proof. According to (2.13)-(2.15), we have the following equations

$$
\begin{cases}\mathscr{B}_{x}^{\alpha} \mathscr{B}_{y}^{\beta} D_{t}^{(2)} E_{i, j}^{n}+\tau^{2} \sigma_{n}^{2} \delta_{x}^{\alpha} \delta_{y}^{\beta} D_{t}^{(2)} E_{i, j}^{n}=\mathscr{B}_{y}^{\beta} \delta_{x}^{\alpha} E_{i, j}^{n}+\mathscr{B}_{x}^{\alpha} \delta_{y}^{\beta} E_{i, j}^{n}+\mathscr{B}_{x}^{\alpha} \mathscr{B}_{y}^{\beta} g_{i, j}^{n}-\mathscr{B}_{x}^{\alpha} \mathscr{B}_{y}^{\beta} \widetilde{g}_{i, j}^{n}, & \left(x_{i}, y_{j}\right) \in \Omega_{h}, 1 \leq n \leq N, \\ E_{i, j}^{n}=0, \quad\left(x_{i}, y_{j}\right) \in \partial \Omega_{h}, 1 \leq n \leq N, & \\ E_{i, j}^{0}=\varphi_{0}\left(x_{i}, y_{j}\right), \quad\left(x_{i}, y_{j}\right) \in \bar{\Omega}_{h} . & \end{cases}
$$

Multiplying by $\tau h_{x} h_{y} E_{i, j}^{n}$ in $(3.1 a)$, then summing from 1 to $M_{1}-1$ on $i$, and summing from 1 to $M_{2}-1$ on $j$, we obtain

$$
\begin{aligned}
\tau\left(\mathscr{B}_{x}^{\alpha} \mathscr{B}_{y}^{\beta} D_{t}^{(2)} E^{n}, E^{n}\right)+\tau\left(\tau^{2} \sigma_{n}^{2} \delta_{x}^{\alpha} \delta_{y}^{\beta} D_{t}^{(2)} E^{n}, E^{n}\right) & =\tau\left(\mathscr{B}_{y}^{\beta} \delta_{x}^{\alpha} E^{n}, E^{n}\right)+\tau\left(\mathscr{B}_{x}^{\alpha} \delta_{y}^{\beta} E^{n}, E^{n}\right) \\
& +\tau h_{x} h_{y} \sum_{i=1}^{M_{1}-1} \sum_{j=1}^{M_{2}-1} \mathscr{B}_{x}^{\alpha} \mathscr{B}_{y}^{\beta}\left(g_{i, j}^{n}-\widetilde{g}_{i, j}^{n}\right) E_{i, j}^{n}
\end{aligned}
$$

where

From Lemma 3.4, we have

$$
\widetilde{g}_{i, j}^{n}= \begin{cases}g\left(x_{i}, y_{j}, t_{1}, \widetilde{u}_{i, j}^{0}\right), & n=1 \\ g\left(x_{i}, y_{j}, t_{n}, 2 \widetilde{u}_{i, j}^{n-1}-\widetilde{u}_{i, j}^{n-2}\right), & n \geq 2\end{cases}
$$

$$
\tau\left(\mathscr{B}_{y}^{\beta} \delta_{x}^{\alpha} E^{n}, E^{n}\right)+\tau\left(\mathscr{B}_{x}^{\alpha} \delta_{y}^{\beta} E^{n}, E^{n}\right) \leq 0 .
$$

Substituting (3.3) into (3.2), it holds that

$$
\tau\left(\mathscr{B}_{x}^{\alpha} \mathscr{B}_{y}^{\beta} D_{t}^{(2)} E^{n}, E^{n}\right)+\tau\left(\tau^{2} \sigma_{n}^{2} \delta_{x}^{\alpha} \delta_{y}^{\beta} D_{t}^{(2)} E^{n}, E^{n}\right) \leq \tau h_{x} h_{y} \sum_{i=1}^{M_{1}-1} \sum_{j=1}^{M_{2}-1} \mathscr{B}_{x}^{\alpha} \mathscr{B}_{y}^{\beta}\left(g_{i, j}^{n}-\widetilde{g}_{i, j}^{n}\right) E_{i, j}^{n} .
$$

Multiplying by $\frac{4}{3}$ in (3.4), and summing from 1 to $k$ on $n$ and replacing $\mathrm{k}$ by $\mathrm{n}$, we get

$$
\begin{aligned}
\frac{4 \tau}{3} \sum_{k=1}^{n}\left(\mathscr{B}_{x}^{\alpha} \mathscr{B}_{y}^{\beta} D_{t}^{(2)} E^{k}, E^{k}\right)+\frac{4 \tau}{3} \sum_{k=1}^{n}\left(\tau^{2} \sigma_{k}^{2} \delta_{x}^{\alpha} \delta_{y}^{\beta} D_{t}^{(2)} E^{k}, E^{k}\right) \\
\quad \leq \frac{4 \tau h_{x} h_{y}}{3} \sum_{k=1}^{n} \sum_{i=1}^{M_{1}-1} \sum_{j=1}^{M_{2}-1} \mathscr{B}_{x}^{\alpha} \mathscr{B}_{y}^{\beta}\left(g_{i, j}^{k}-\widetilde{g}_{i, j}^{k}\right) E_{i, j}^{k} .
\end{aligned}
$$

According to Lemma 3.1, we have

$$
\begin{aligned}
\frac{4 \tau}{3} \sum_{k=1}^{n}\left(\mathscr{B}_{x}^{\alpha} \mathscr{B}_{y}^{\beta} D_{t}^{(2)} E^{k}, E^{k}\right) & =\frac{4 \tau}{3} \sum_{k=1}^{n}\left(D_{t}^{(2)} Q_{x} Q_{y} E^{k}, Q_{x} Q_{y} E^{k}\right) \\
& \geq\left\|E^{n}\right\|_{\mathscr{B}}^{2}-\frac{1}{3}\left\|E^{n-1}\right\|_{\mathscr{B}}^{2}-\frac{1}{3}\left\|E^{1}\right\|_{\mathscr{B}}^{2}-\frac{1}{3}\left\|E^{0}\right\|_{\mathscr{B}}^{2},
\end{aligned}
$$

and

$$
\begin{aligned}
\frac{4 \tau}{3} \sum_{k=1}^{n} \sigma_{k}^{2}\left(\delta_{x}^{\alpha} \delta_{y}^{\beta} D_{t}^{(2)} E^{k}, E^{k}\right)= & \frac{4 \tau}{3} \sum_{k=1}^{n} \sigma_{k}^{2}\left(D_{t}^{(2)} \Lambda_{x} \Lambda_{y} E^{k}, \Lambda_{x} \Lambda_{y} E^{k}\right) \\
= & \frac{4}{9} \cdot \frac{4 \tau}{3} \sum_{k=2}^{n}\left(D_{t}^{(2)} \Lambda_{x} \Lambda_{y} E^{k}, \Lambda_{x} \Lambda_{y} E^{k}\right) \\
& +\frac{4 \tau}{3}\left(D_{t}^{(2)} \Lambda_{x} \Lambda_{y} E^{1}, \Lambda_{x} \Lambda_{y} E^{1}\right) \\
\geq & \frac{4}{9}\left\|E^{n}\right\|_{\delta}^{2}-\frac{4}{27}\left\|E^{n-1}\right\|_{\delta}^{2}-\frac{4}{9}\left\|E^{1}\right\|_{\delta}^{2}+\frac{4}{27}\left\|E^{0}\right\|_{\delta}^{2}-\frac{8}{27}\left\|E^{1}-E^{0}\right\|_{\delta}^{2} \\
& +\frac{2}{3}\left\|E^{1}\right\|_{\delta}^{2}-\frac{2}{3}\left\|E^{0}\right\|_{\delta}^{2}+\frac{2}{3}\left\|E^{1}-E^{0}\right\|_{\delta}^{2} \\
\geq & \frac{4}{9}\left\|E^{n}\right\|_{\delta}^{2}-\frac{4}{27}\left\|E^{n-1}\right\|_{\delta}^{2}-\frac{14}{27}\left\|E^{0}\right\|_{\delta}^{2} .
\end{aligned}
$$


Substituting (3.6)-(3.7) into (3.5), we obtain

$$
\begin{aligned}
\left\|E^{n}\right\|_{\mathscr{B}}^{2}+\frac{4}{9} \tau^{2}\left\|E^{n}\right\|_{\delta}^{2} \leq & \frac{1}{3}\left(\left\|E^{n-1}\right\|_{\mathscr{B}}^{2}+\frac{4}{9} \tau^{2}\left\|E^{n-1}\right\|_{\delta}^{2}\right)+\frac{1}{3}\left\|E^{1}\right\|_{\mathscr{B}}^{2}+\frac{1}{3}\left(\left\|E^{0}\right\|_{\mathscr{B}}^{2}+\frac{14}{9} \tau^{2}\left\|E^{0}\right\|_{\delta}^{2}\right) \\
& +\frac{4 \tau h_{x} h_{y}}{3} \sum_{k=1}^{n} \sum_{i=1}^{M_{1}-1} \sum_{j=1}^{M_{2}-1}\left|\mathscr{B}_{x}^{\alpha} \mathscr{B}_{y}^{\beta}\left(g_{i, j}^{k}-\widetilde{g}_{i, j}^{k}\right) E_{i, j}^{k}\right| .
\end{aligned}
$$

When $k=1$, from Lemma 3.2, (1.4) and Cauchy-Schwarz inequality, we have

$$
\begin{aligned}
h_{x} h_{y} \sum_{i=1}^{M_{1}-1} \sum_{j=1}^{M_{2}-1} \mid \mathscr{B}_{x}^{\alpha} \mathscr{B}_{y}^{\beta}\left(g_{i, j}^{1}-\widetilde{g}_{i, j}^{1}\right) & E_{i, j}^{1} \mid \leq\left(\mathscr{B}_{x}^{\alpha} \mathscr{B}_{y}^{\beta}\left|g^{1}-\widetilde{g}^{1}\right|,\left|E^{1}\right|\right) \\
& \leq L\left(\mathscr{B}_{x}^{\alpha} \mathscr{B}_{y}^{\beta}\left|E^{0}\right|,\left|E^{1}\right|\right) \\
& =L\left(Q_{x} Q_{y}\left|E^{0}\right|, Q_{x} Q_{y}\left|E^{1}\right|\right) \\
& \leq \frac{L}{2}\left\|\left|E^{0}\right|\right\|_{\mathscr{B}}^{2}+\frac{L}{2}\left\|\mid E^{1}\right\| \|_{\mathscr{B}}^{2} \\
& \leq \frac{L}{2}\left\|\left|E^{0}\right|\right\|^{2}+\frac{L}{2}\left\|\mid E^{1}\right\| \|^{2} \\
& =\frac{L}{2}\left\|E^{0}\right\|^{2}+\frac{L}{2}\left\|E^{1}\right\|^{2}
\end{aligned}
$$

When $k \geq 2$, according to Lemma 3.2, (1.4) and Cauchy-Schwarz inequality, we get

$$
\begin{aligned}
h_{x} h_{y} \sum_{i=1}^{M_{1}-1} \sum_{j=1}^{M_{2}-1} \mid \mathscr{B}_{x}^{\alpha} \mathscr{B}_{y}^{\beta}\left(g_{i, j}^{k}\right. & \left.-\widetilde{g}_{i, j}^{k}\right) E_{i, j}^{k} \mid \leq\left(\mathscr{B}_{x}^{\alpha} \mathscr{B}_{y}^{\beta}\left|g^{k}-\widetilde{g}^{k}\right|,\left|E^{k}\right|\right) \\
& \leq L\left(\mathscr{B}_{x}^{\alpha} \mathscr{B}_{y}^{\beta}\left|2 E^{k-1}-E^{k-2}\right|,\left|E^{k}\right|\right) \\
& \leq 2 L\left(\mathscr{B}_{x}^{\alpha} \mathscr{B}_{y}^{\beta}\left|E^{k-1}\right|,\left|E^{k}\right|\right)+L\left(\mathscr{B}_{x}^{\alpha} \mathscr{B}_{y}^{\beta}\left|E^{k-2}\right|,\left|E^{k}\right|\right) \\
& \leq 2 L\left\|\left|E^{k-1}\right|\right\|_{\mathscr{B}}\left\|\left|E^{k}\right|\right\|_{\mathscr{B}}+L\left\|\left|E^{k-2}\right|\right\|_{\mathscr{B}}\left\|\left|E^{k}\right|\right\| \mathscr{B}_{\mathscr{B}} \\
& \leq L\left\|\left|E^{k-1}\right|\right\|_{\mathscr{B}}^{2}+L\left\|\left|E^{k}\right|\right\|_{\mathscr{B}}^{2}+\frac{L}{2}\left\|\left|E^{k-2}\right|\right\|_{\mathscr{B}}^{2}+\frac{L}{2}\left\|\mid E^{k}\right\|_{\mathscr{B}}^{2} \\
& =\frac{3 L}{2}\left\|\left|E^{k}\|\|_{\mathscr{B}}^{2}+L\left\|\left|E^{k-1}\right|\right\|_{\mathscr{B}}^{2}+\frac{L}{2}\left\|\left|E^{k-2}\right|\right\|_{\mathscr{B}}^{2}\right.\right. \\
& \leq \frac{3 L}{2}\left\|\left|E^{k}\right|\right\|^{2}+L\left\|\left|E^{k-1}\right|\right\|^{2}+\frac{L}{2}\left\|\left|E^{k-2}\right|\right\|^{2} \\
& =\frac{3 L}{2}\left\|E^{k}\right\|^{2}+L\left\|E^{k-1}\right\|^{2}+\frac{L}{2}\left\|E^{k-2}\right\|^{2} .
\end{aligned}
$$

Substituting (3.9)-(3.10) into (3.8), from Lemma 3.2, we have

$$
\begin{aligned}
\left\|E^{n}\right\|_{\mathscr{B}}^{2} \leq & \left\|E^{n}\right\|_{\mathscr{B}}^{2}+\frac{4}{9} \tau^{2}\left\|E^{n}\right\|_{\delta}^{2} \\
\leq & \frac{1}{3}\left(\left\|E^{n-1}\right\|_{\mathscr{B}}^{2}+\frac{4}{9} \tau^{2}\left\|E^{n-1}\right\|_{\delta}^{2}\right)+\frac{1}{3}\left\|E^{1}\right\|_{\mathscr{B}}^{2}+\frac{1}{3}\left(\left\|E^{0}\right\|_{\mathscr{B}}^{2}+\frac{14}{9} \tau^{2}\left\|E^{0}\right\|_{\delta}^{2}\right) \\
& +\frac{4 \tau L}{3} \sum_{k=2}^{n}\left(\frac{3}{2}\left\|E^{k}\right\|^{2}+\left\|E^{k-1}\right\|^{2}+\frac{1}{2}\left\|E^{k-2}\right\|^{2}\right) \\
& +\frac{2 \tau L}{3}\left\|E^{1}\right\|^{2}+\frac{2 \tau L}{3}\left\|E^{0}\right\|^{2} \\
\leq & \frac{1}{3}\left(\left\|E^{n-1}\right\|_{\mathscr{B}}^{2}+\frac{4}{9} \tau^{2}\left\|E^{n-1}\right\|_{\delta}^{2}\right)+\frac{1}{3}\left\|E^{1}\right\|_{\mathscr{B}}^{2}+\frac{1}{3}\left(\left\|E^{0}\right\|_{\mathscr{B}}^{2}+\frac{14}{9} \tau^{2}\left\|E^{0}\right\|_{\delta}^{2}\right) \\
& +4 \tau L \sum_{k=2}^{n}\left(\frac{3}{2}\left\|E^{k}\right\|_{\mathscr{B}}^{2}+\left\|E^{k-1}\right\|_{\mathscr{B}}^{2}+\frac{1}{2}\left\|E^{k-2}\right\|_{\mathscr{B}}^{2}\right) \\
& +2 \tau L\left\|E^{1}\right\|_{\mathscr{B}}^{2}+2 \tau L\left\|E^{0}\right\|_{\mathscr{B}}^{2} .
\end{aligned}
$$

Taking $n=1$ in (3.11), we find

$$
\left\|E^{1}\right\|_{\mathscr{B}}^{2} \leq\left\|E^{0}\right\|_{\mathscr{B}}^{2}+\tau^{2}\left\|E^{0}\right\|_{\delta}^{2}+3 \tau L\left\|E^{1}\right\|_{\mathscr{B}}^{2}+3 \tau L\left\|E^{0}\right\|_{\mathscr{B}}^{2}
$$

Substituting (3.12) into (3.11), we obtain

$$
\begin{aligned}
\left\|E^{n}\right\|_{\mathscr{B}}^{2}+\frac{4}{9} \tau^{2}\left\|E^{n}\right\|_{\delta}^{2} \leq \frac{1}{3} & \left.\left\|E^{n-1}\right\|_{\mathscr{B}}^{2}+\frac{4}{9} \tau^{2}\left\|E^{n-1}\right\|_{\delta}^{2}\right)+\frac{2}{3}\left(\left\|E^{0}\right\|_{\mathscr{B}}^{2}+\frac{23}{18} \tau^{2}\left\|E^{0}\right\|_{\delta}^{2}\right) \\
& +3 \tau L\left\|E^{1}\right\|_{\mathscr{B}}^{2}+3 \tau L\left\|E^{0}\right\|_{\mathscr{B}}^{2} \\
& +4 \tau L \sum_{k=2}^{n}\left(\frac{3}{2}\left\|E^{k}\right\|_{\mathscr{B}}^{2}+\left\|E^{k-1}\right\|_{\mathscr{B}}^{2}+\frac{1}{2}\left\|E^{k-2}\right\|_{\mathscr{B}}^{2}\right)
\end{aligned}
$$

Taking $0 \leq n_{0} \leq n$ such that

$$
\left\|E^{n_{0}}\right\|_{\mathscr{B}}^{2}+\frac{4}{9} \tau^{2}\left\|E^{n_{0}}\right\|_{\delta}^{2}=\max _{0 \leq l \leq n}\left(\left\|E^{l}\right\|_{\mathscr{B}}^{2}+\frac{4}{9} \tau^{2}\left\|E^{l}\right\|_{\delta}^{2}\right) \geq\left\|E^{l}\right\|_{\mathscr{B}}^{2}, \quad 0 \leq l \leq n .
$$


Therefore

$$
\frac{1}{3}\left(\left\|E^{n-1}\right\|_{\mathscr{B}}^{2}+\frac{4}{9} \tau^{2}\left\|E^{n-1}\right\|_{\delta}^{2}\right) \leq \frac{1}{3}\left(\left\|E^{n_{0}}\right\|_{\mathscr{B}}^{2}+\frac{4}{9} \tau^{2}\left\|E^{n_{0}}\right\|_{\delta}^{2}\right) .
$$

According to Lemma 3.2 and Cauchy-Schwarz inequality, it follows from (3.13)-(3.14) that

$$
\begin{aligned}
\left\|E^{n_{0}}\right\|_{\mathscr{B}}^{2}+\frac{4}{9} \tau^{2}\left\|E^{n_{0}}\right\|_{\delta}^{2} \leq & \left(\left\|E^{0}\right\|_{\mathscr{B}}^{2}+\frac{23}{18} \tau^{2}\left\|E^{0}\right\|_{\delta}^{2}\right)+\frac{9}{2} \tau L\left\|E^{1}\right\|_{\mathscr{B}}^{2}+\frac{9}{2} \tau L\left\|E^{0}\right\|_{\mathscr{B}}^{2} \\
& +6 \tau L \sum_{k=2}^{n_{0}}\left(\frac{3}{2}\left\|E^{k}\right\|_{\mathscr{B}}^{2}+\left\|E^{k-1}\right\|_{\mathscr{B}}^{2}+\frac{1}{2}\left\|E^{k-2}\right\|_{\mathscr{B}}^{2}\right) \\
\leq & \left(\left\|E^{0}\right\|^{2}+\frac{23}{18} \tau^{2}\left\|E^{0}\right\|_{\delta}^{2}\right)+\frac{9}{2} \tau L\left\|E^{1}\right\|_{\mathscr{B}}^{2}+\frac{9}{2} \tau L\left\|E^{0}\right\|_{\mathscr{B}}^{2} \\
& +6 \tau L \sum_{k=2}^{n}\left(\frac{3}{2}\left\|E^{k}\right\|_{\mathscr{B}}^{2}+\left\|E^{k-1}\right\|_{\mathscr{B}}^{2}+\frac{1}{2}\left\|E^{k-2}\right\|_{\mathscr{B}}^{2}\right) \\
= & \left(\left(I+\frac{23}{18} \tau^{2} \delta_{x}^{\alpha} \delta_{y}^{\beta}\right) E^{0}, E^{0}\right)+\frac{9}{2} \tau L\left\|E^{1}\right\|_{\mathscr{B}}^{2}+\frac{9}{2} \tau L\left\|E^{0}\right\|_{\mathscr{B}}^{2} \\
& +6 \tau L \sum_{k=2}^{n}\left(\frac{3}{2}\left\|E^{k}\right\|_{\mathscr{B}}^{2}+\left\|E^{k-1}\right\|_{\mathscr{B}}^{2}+\frac{1}{2}\left\|E^{k-2}\right\|_{\mathscr{B}}^{2}\right) \\
\leq & \sqrt{3}\left\|\left(I+\frac{23}{18} \tau^{2} \delta_{x}^{\alpha} \delta_{y}^{\beta}\right) E^{0}\right\|\left\|E^{0}\right\|_{\mathscr{B}}+\frac{9}{2} \tau L\left\|E^{1}\right\|_{\mathscr{B}}^{2}+\frac{9}{2} \tau L\left\|E^{0}\right\|_{\mathscr{B}}^{2} \\
& +6 \tau L \sum_{k=2}^{n}\left(\frac{3}{2}\left\|E^{k}\right\|_{\mathscr{B}}^{2}+\left\|E^{k-1}\right\|_{\mathscr{B}}^{2}+\frac{1}{2}\left\|E^{k-2}\right\|_{\mathscr{B}}^{2}\right) .
\end{aligned}
$$

We obtain from (3.14) and Lemma 3.2 that

$$
\begin{aligned}
\left\|E^{n_{0}}\right\|_{\mathscr{B}}^{2}+\frac{4}{9} \tau^{2}\left\|E^{n_{0}}\right\|_{\delta}^{2} \leq & \sqrt{3}\left\|\left(I+\frac{23}{18} \tau^{2} \delta_{x}^{\alpha} \delta_{y}^{\beta}\right) E^{0}\right\|\left\|E^{0}\right\|_{\mathscr{B}}+\frac{9}{2} \tau L\left\|E^{1}\right\|_{\mathscr{B}}^{2}+\frac{9}{2} \tau L\left\|E^{0}\right\|_{\mathscr{B}}^{2} \\
& +6 \tau L \sum_{k=2}^{n}\left(\frac{3}{2}\left\|E^{k}\right\|_{\mathscr{B}}^{2}+\left\|E^{k-1}\right\|_{\mathscr{B}}^{2}+\frac{1}{2}\left\|E^{k-2}\right\|_{\mathscr{B}}^{2}\right) \\
\leq & \left(\sqrt{3}\left\|\left(I+\frac{23}{18} \tau^{2} \delta_{x}^{\alpha} \delta_{y}^{\beta}\right) E^{0}\right\|+\frac{9}{2} \tau L\left\|E^{1}\right\| \mathscr{B}+\frac{9}{2} \tau L\left\|E^{0}\right\|_{\mathscr{B}}\right. \\
& \left.+6 \tau L \sum_{k=2}^{n}\left(\frac{3}{2}\left\|E^{k}\right\|_{\mathscr{B}}+\left\|E^{k-1}\right\|_{\mathscr{B}}+\frac{1}{2}\left\|E^{k-2}\right\|_{\mathscr{B}}\right)\right) \sqrt{\left\|E^{n_{0}}\right\|_{\mathscr{B}}^{2}+\frac{4}{9} \tau^{2}\left\|E^{n_{0}}\right\|_{\delta}^{2}}
\end{aligned}
$$

Thus, it follows from (3.14) and (3.16) that

$$
\begin{aligned}
\left\|E^{n}\right\|_{\mathscr{B}} \leq & \sqrt{\left\|E^{n_{0}}\right\|_{\mathscr{B}}^{2}+\frac{4}{9} \tau^{2}\left\|E^{n_{0}}\right\|_{\delta}^{2}} \\
\leq & \sqrt{3}\left\|\left(I+\frac{23}{1} \tau^{2} \delta_{x}^{\alpha} \delta_{y}^{\beta}\right) E^{0}\right\|+\frac{9}{2} \tau L\left\|E^{1}\right\|_{\mathscr{B}}+\frac{9}{2} \tau L\left\|E^{0}\right\|_{\mathscr{B}} \\
& +6 \tau L \sum_{k=2}^{n}\left(\frac{3}{2}\left\|E^{k}\right\|_{\mathscr{B}}+\left\|E^{k-1}\right\|_{\mathscr{B}}+\frac{1}{2}\left\|E^{k-2}\right\|_{\mathscr{B}}\right) \\
= & \sqrt{3}\left\|\left(I+\frac{23}{18} \tau^{2} \delta_{x}^{\alpha} \delta_{y}^{\beta}\right) E^{0}\right\|+9 \tau L \sum_{k=0}^{n-1}\left\|E^{k}\right\|_{\mathscr{B}}+6 \tau L \sum_{k=0}^{n-1}\left\|E^{k}\right\|_{\mathscr{B}}+3 \tau L \sum_{k=0}^{n-1}\left\|E^{k}\right\|_{\mathscr{B}} \\
& +9 \tau L\left\|E^{n}\right\|_{\mathscr{B}}-\frac{9}{2} \tau L\left\|E^{1}\right\|_{\mathscr{B}}-\frac{21}{2} \tau L\left\|E^{0}\right\|_{\mathscr{B}}-3 \tau L\left\|E^{n-1}\right\|_{\mathscr{B}} \\
\leq & \sqrt{3}\left\|\left(I+\frac{23}{18} \tau^{2} \delta_{x}^{\alpha} \delta_{y}^{\beta}\right) E^{0}\right\|+18 \tau L \sum_{k=0}^{n-1}\left\|E^{k}\right\|_{\mathscr{B}}+9 \tau L\left\|E^{n}\right\|_{\mathscr{B}} .
\end{aligned}
$$

we can obtain the recursion from (3.17) that

$$
(1-9 \tau L)\left\|E^{n}\right\|_{\mathscr{B}} \leq \sqrt{3}\left\|\left(I+\frac{23}{18} \tau^{2} \delta_{x}^{\alpha} \delta_{y}^{\beta}\right) E^{0}\right\|+18 \tau L \sum_{k=0}^{n-1}\left\|E^{k}\right\|_{\mathscr{B}}
$$

For any $v \in(0,1)$, and $0<\tau \leq \tau_{0}=\frac{1-v}{9 L}$, according to Lemma 3.5, it follows from (3.18) that

$$
\begin{aligned}
\left\|E^{n}\right\|_{\mathscr{B}} & \leq \frac{\sqrt{3}}{v}\left\|\left(I+\frac{23}{18} \tau^{2} \delta_{x}^{\alpha} \delta_{y}^{\beta}\right) E^{0}\right\|+\frac{18}{v} \tau L \sum_{k=0}^{n-1}\left\|E^{k}\right\|_{\mathscr{B}} \\
& \leq \frac{\sqrt{3}}{v}\left\|\left(I+\frac{23}{18} \tau^{2} \delta_{x}^{\alpha} \delta_{y}^{\beta}\right) E^{0}\right\| e^{\frac{18}{v} \tau L n} \\
& \leq \frac{\sqrt{3}}{v}\left\|\left(I+\frac{23}{18} \tau^{2} \delta_{x}^{\alpha} \delta_{y}^{\beta}\right) E^{0}\right\| e^{\frac{18}{v}} L T .
\end{aligned}
$$

Recalling Lemma 3.2, we have

$$
\left\|E^{n}\right\| \leq \frac{3}{v} e^{\frac{18}{v} L T}\left\|\left(I+\frac{23}{18} \tau^{2} \delta_{x}^{\alpha} \delta_{y}^{\beta}\right) E^{0}\right\|, \quad n \geq 1 .
$$

Therefore, the numerical scheme is stable. The proof is completed. 
Theorem 3.2. For any positive real number $v \in(0,1)$, if $0<\tau \leq \tau_{0}=\frac{1-v}{9 L}$, then the numerical scheme (2.13)-(2.15) is convergent, and it holds that

$$
\max _{1 \leq n \leq N}\left\|\eta^{n}\right\|=O\left(\tau^{2}+h_{x}^{4}+h_{y}^{4}\right) .
$$

Proof. Denote $\eta_{i, j}^{n}=u\left(x_{i}, y_{j}, t_{n}\right)-u_{i, j}^{n},\left(x_{i}, y_{j}\right) \in \Omega_{h}, 0 \leq n \leq N$, and $\eta^{n}=\left(\eta_{0,0}^{n}, \cdots, \eta_{M_{1}, 0}^{n}, \cdots, \eta_{0, M_{2}}^{n}, \cdots, \eta_{M_{1}, M_{2}}^{n}\right)$. Similarly with the inference procedure of Theorem 3.1, it follows from (3.13) that

$$
\begin{aligned}
\left\|\eta^{n}\right\|_{\mathscr{B}}^{2}+\frac{4}{9} \tau^{2}\left\|\eta^{n}\right\|_{\delta}^{2} \leq & \frac{1}{3}\left(\left\|\eta^{n-1}\right\|_{\mathscr{B}}^{2}+\frac{4}{9} \tau^{2}\left\|\eta^{n-1}\right\|_{\delta}^{2}\right)+\frac{2}{3}\left(\left\|\eta^{0}\right\|_{\mathscr{B}}^{2}+\frac{23}{18} \tau^{2}\left\|\eta^{0}\right\|_{\delta}^{2}\right) \\
& +3 \tau L\left\|\eta^{1}\right\|_{\mathscr{B}}^{2}+3 \tau L\left\|\eta^{0}\right\|_{\mathscr{B}}^{2} \\
& +4 \tau L \sum_{k=2}^{n}\left(\frac{3}{2}\left\|\eta^{k}\right\|_{\mathscr{B}}^{2}+\left\|\eta^{k-1}\right\|_{\mathscr{B}}^{2}+\frac{1}{2}\left\|\eta^{k-2}\right\|_{\mathscr{B}}^{2}\right) \\
& +\frac{4 \tau h_{x} h_{y}}{3} \sum_{k=2}^{n} \sum_{i=1}^{M_{1}-1} \sum_{j=1}^{M_{2}-1} \mathscr{R}_{i, j}^{k} \eta_{i, j}^{k}+2 \tau h_{x} h_{y} \sum_{i=1}^{M_{1}-1} \sum_{j=1}^{M_{2}-1} \mathscr{R}_{i, j}^{1} \eta_{i, j}^{1} \\
\leq & \frac{1}{3}\left(\left\|\eta^{n-1}\right\|_{\mathscr{B}}^{2}+\frac{4}{9} \tau^{2}\left\|\eta^{n-1}\right\|_{\delta}^{2}\right)+\frac{2}{3}\left(\left\|\eta^{0}\right\|_{\mathscr{B}}^{2}+\frac{23}{18} \tau^{2}\left\|\eta^{0}\right\|_{\delta}^{2}\right) \\
& +3 \tau L\left\|\eta^{1}\right\|_{\mathscr{B}}^{2}+3 \tau L\left\|\eta^{0}\right\|_{\mathscr{B}}^{2} \\
& +4 \tau L \sum_{k=2}^{n}\left(\frac{3}{2}\left\|\eta^{k}\right\|_{\mathscr{B}}^{2}+\left\|\eta^{k-1}\right\|_{\mathscr{B}}^{2}+\frac{1}{2}\left\|\eta^{k-2}\right\|_{\mathscr{B}}^{2}\right) \\
& +\frac{4 \sqrt{3} \tau}{3} \sum_{k=2}^{n}\left\|\mathscr{R}^{k}\right\|\left\|\eta^{k}\right\|_{\mathscr{B}}+2 \sqrt{3} \tau\left\|\mathscr{R}^{1}\right\|\left\|\eta^{1}\right\| \|_{\mathscr{B}} .
\end{aligned}
$$

Similarly with (3.14)-(3.17), we obtain

$$
(1-9 \tau L)\left\|\eta^{n}\right\|_{\mathscr{B}} \leq 2 \sqrt{3} \tau \sum_{k=2}^{n}\left\|\mathscr{R}^{k}\right\|+3 \sqrt{3} \tau\left\|\mathscr{R}^{1}\right\|+18 \tau L \sum_{k=0}^{n-1}\left\|\eta^{k}\right\|_{\mathscr{B}}
$$

Assume that $\tau \leq 1$, it follows from (2.12) and the definition of the norm that

$$
\begin{aligned}
& \tau\left\|\mathscr{R}^{1}\right\| \leq c_{1} \sqrt{(b-a)(d-c)}\left(\tau^{2}+\tau h_{x}^{4}+\tau h_{y}^{4}\right) \leq c_{1} \sqrt{(b-a)(d-c)}\left(\tau^{2}+h_{x}^{4}+h_{y}^{4}\right) \\
& =c_{1}^{*}\left(\tau^{2}+h_{x}^{4}+h_{y}^{4}\right) \text {, } \\
& \tau \sum_{k=2}^{n-1}\left\|\mathscr{R}^{k}\right\| \leq \tau(n-2) c_{2} \sqrt{(b-a)(d-c)}\left(\tau^{2}+h_{x}^{4}+h_{y}^{4}\right) \leq c_{2} T \sqrt{(b-a)(d-c)}\left(\tau^{2}+h_{x}^{4}+h_{y}^{4}\right) \\
& =c_{2}^{*}\left(\tau^{2}+h_{x}^{4}+h_{y}^{4}\right) \text {, }
\end{aligned}
$$

where $c_{1}^{*}=c_{1} \sqrt{(b-a)(d-c)}, c_{2}^{*}=c_{2} T \sqrt{(b-a)(d-c)}$.

For any positive real number $v \in(0,1)$, let $\tau_{0}=\frac{1-v}{9 L}$, if $0<\tau \leq \tau_{0}$, it follows from Lemma 3.5 and (3.21) that

$$
\begin{aligned}
\frac{\sqrt{3}}{3}\left\|\eta^{n}\right\| & \leq\left\|\eta^{n}\right\|_{\mathscr{B}} \leq \frac{2 \sqrt{3} \tau}{v} \sum_{k=2}^{n}\left\|\mathscr{R}^{k}\right\|+\frac{3 \sqrt{3} \tau}{v}\left\|\mathscr{R}^{1}\right\|+\frac{18 \tau L}{v} \sum_{k=0}^{n-1}\left\|\eta^{k}\right\|_{\mathscr{B}} \\
& \leq\left(\frac{2 \sqrt{3} \tau}{v} \sum_{k=2}^{n}\left\|\mathscr{R}^{k}\right\|+\frac{3 \sqrt{3} \tau}{v}\left\|\mathscr{R}^{1}\right\|\right) e^{\frac{18 n \tau L}{v}} \\
& \leq c_{3}\left(\tau^{2}+h_{x}^{4}+h_{y}^{4}\right),
\end{aligned}
$$

where $c_{3}=\frac{\sqrt{3}\left(2 c_{1}^{*}+3 c_{2}^{*}\right)}{v} e^{\frac{18 L T}{v}}$.

Thus

$$
\max _{1 \leq n \leq N}\left\|\eta^{n}\right\|=O\left(\tau^{2}+h_{x}^{4}+h_{y}^{4}\right)
$$

The proof is completed.

\section{Numerical experiments}

Let $\|\eta(h, \tau)\|=\sqrt{h^{2} \sum_{i=1}^{M_{1}-1} \sum_{j=1}^{M_{2}-1}\left|u\left(x_{i}, y_{j}, t_{N}\right)-u_{i, j}^{N}\right|^{2}}$ and $\|\eta(h, \tau)\|_{\infty}=\max _{\substack{1 \leq i \leq M_{1}-1 \\ 1 \leq j \leq M_{2}-1}}\left|u\left(x_{i}, y_{j}, t_{N}\right)-u_{i, j}^{N}\right|$ denote as $L_{2}$ norm and maximum norm errors with $h=h_{x}=h_{y}$ at $t=t_{N}$, respectively. The observation orders of $L_{2}$ norm and maximum norm are defined by

$$
\text { Rate }_{\tau}=\log _{2}\left(\frac{\|\eta(h, 2 \tau)\|}{\|\eta(h, \tau)\|}\right), \quad \text { Rate }_{h}=\log _{2}\left(\frac{\|\eta(2 h, 4 \tau)\|}{\|\eta(h, \tau)\|}\right) .
$$




$$
\text { Rate }_{\tau}^{\infty}=\log _{2}\left(\frac{\|\eta(h, 2 \tau)\|_{\infty}}{\|\eta(h, \tau)\|_{\infty}}\right), \quad \text { Rate }_{h}^{\infty}=\log _{2}\left(\frac{\|\eta(2 h, 4 \tau)\|_{\infty}}{\|\eta(h, \tau)\|_{\infty}}\right) .
$$

Example 4.1. Consider the following two-dimensional Riesz space fractional nonlinear reaction-diffusion equation

$$
\frac{\partial u(x, y, t)}{\partial t}=\kappa_{1} \frac{\partial^{\alpha} u(x, y, t)}{\partial|x|^{\alpha}}+\kappa_{2} \frac{\partial^{\alpha} u(x, y, t)}{\partial|y|^{\alpha}}+g(x, y, t, u(x, y, t)), \quad 0<x, y<1,0<t \leq 1,
$$

with boundary and initial conditions

$$
\begin{gathered}
u(0, y, t)=u(x, 0, t)=0, \quad u(x, 1, t)=u(1, y, t)=0, \quad 0 \leq x, y \leq 1,0<t \leq 1, \\
u(x, y, 0)=x^{4}(1-x)^{4} y^{4}(1-y)^{4}, \quad 0 \leq x, y \leq 1,
\end{gathered}
$$

where $1<\alpha, \beta<2$, the nonlinear source term $g(x, y, t, u(x, y, t))$ is

$$
\begin{aligned}
g(x, y, t, u(x, y, t))= & (u(x, t))^{2}-e^{-t} y^{4}(1-y)^{4}\left(x^{4}(1-x)^{4}+\frac{\Gamma(5)}{\Gamma(5-\alpha)}\left\{\kappa_{1} c_{\alpha} x^{4-\alpha}+\kappa_{1} c_{\alpha}(1-x)^{4-\alpha}\right\}\right. \\
- & 4 \frac{\Gamma(6)}{\Gamma(6-\alpha)}\left\{\kappa_{1} c_{\alpha} x^{5-\alpha}+\kappa_{1} c_{\alpha}(1-x)^{5-\alpha}\right\}+6 \frac{\Gamma(7)}{\Gamma(7-\alpha)}\left\{\kappa_{1} c_{\alpha} x^{6-\alpha}+\kappa_{1} c_{\alpha}(1-x)^{6-\alpha}\right\} \\
& \left.-4 \frac{\Gamma(8)}{\Gamma(8-\alpha)}\left\{\kappa_{1} c_{\alpha} x^{7-\alpha}+\kappa_{1} c_{\alpha}(1-x)^{7-\alpha}\right\}+\frac{\Gamma(9)}{\Gamma(9-\alpha)}\left\{\kappa_{1} c_{\alpha} x^{8-\alpha}+\kappa_{1} c_{\alpha}(1-x)^{8-\alpha}\right\}\right) \\
& -e^{-t} x^{4}(1-x)^{4}\left(e^{-t} x^{4}(1-x)^{4} y^{8}(1-y)^{8}+\frac{\Gamma(5)}{\Gamma(5-\beta)}\left\{\kappa_{2} c_{\beta} y^{4-\beta}+\kappa_{2} c_{\beta}(1-y)^{4-\beta}\right\}\right. \\
& -4 \frac{\Gamma(6)}{\Gamma(6-\beta)}\left\{\kappa_{2} c_{\beta} y^{5-\beta}+\kappa_{2} c_{\beta}(1-y)^{5-\beta}\right\}+6 \frac{\Gamma(7)}{\Gamma(7-\beta)}\left\{\kappa_{2} c_{\beta} y^{6-\beta}+\kappa_{2} c_{\beta}(1-y)^{6-\beta}\right\} \\
& \left.-4 \frac{\Gamma(8)}{\Gamma(8-\beta)}\left\{\kappa_{2} c_{\beta} y^{7-\beta}+\kappa_{2} c_{\beta}(1-y)^{7-\beta}\right\}+\frac{\Gamma(9)}{\Gamma(9-\beta)}\left\{\kappa_{2} c_{\beta} y^{8-\beta}+\kappa_{2} c_{\beta}(1-y)^{8-\beta}\right\}\right) .
\end{aligned}
$$

The exact solution of the problem $(4.1 a)-(4.1 \mathrm{c})$ is

$$
u(x, y, t)=e^{-t} x^{4}(1-x)^{4} y^{4}(1-y)^{4} .
$$

Table 4.1: Errors and corresponding spatial observation orders of BCIM for $\kappa_{1}=2, \kappa_{2}=4$.

\begin{tabular}{cccccccc}
\hline$\alpha$ & $\beta$ & $h$ & $\tau$ & $\|\eta(h, \tau)\|_{\infty}$ & Rate $_{h}^{\infty}$ & $\|\eta(h, \tau)\|$ & Rate $_{h}$ \\
\hline \multirow{2}{*}{1.1} & \multirow{2}{*}{1.5} & $\frac{1}{8}$ & $\frac{1}{64}$ & $2.3306 \mathrm{e}-08$ & $*$ & $9.3070 \mathrm{e}-09$ & $*$ \\
& & $\frac{1}{16}$ & $\frac{1}{256}$ & $1.3729 \mathrm{e}-09$ & 4.085 & $5.4995 \mathrm{e}-10$ & 4.081 \\
& & $\frac{1}{32}$ & $\frac{1}{1024}$ & $8.1699 \mathrm{e}-11$ & 4.071 & $3.1847 \mathrm{e}-11$ & 4.110 \\
& & $\frac{1}{64}$ & $\frac{1}{4096}$ & $4.7570 \mathrm{e}-12$ & 4.102 & $1.8390 \mathrm{e}-12$ & 4.114 \\
\hline \hline \multirow{2}{*}{1.3} & \multirow{2}{*}{1.7} & $\frac{1}{8}$ & $\frac{1}{64}$ & $2.8704 \mathrm{e}-08$ & $*$ & $1.1769 \mathrm{e}-08$ & $*$ \\
& & $\frac{1}{16}$ & $\frac{1}{256}$ & $1.7359 \mathrm{e}-9$ & 4.048 & $7.1648 \mathrm{e}-10$ & 4.038 \\
& & $\frac{1}{32}$ & $\frac{1}{1024}$ & $1.0488 \mathrm{e}-10$ & 4.049 & $4.2172 \mathrm{e}-11$ & 4.087 \\
& & $\frac{1}{64}$ & $\frac{1}{4096}$ & $5.9342 \mathrm{e}-12$ & 4.144 & $2.3909 \mathrm{e}-12$ & 4.141 \\
\hline \hline \multirow{2}{*}{1.5} & \multirow{2}{*}{1.9} & $\frac{1}{8}$ & $\frac{1}{64}$ & $3.4947 \mathrm{e}-08$ & $*$ & $1.4841 \mathrm{e}-08$ & $*$ \\
& & $\frac{1}{16}$ & $\frac{1}{256}$ & $2.1909 \mathrm{e}-09$ & 3.996 & $9.4251 \mathrm{e}-10$ & 3.977 \\
& & $\frac{1}{32}$ & $\frac{1}{1024}$ & $1.3642 \mathrm{e}-10$ & 4.005 & $5.7485 \mathrm{e}-11$ & 4.035 \\
& & $\frac{1}{64}$ & $\frac{1}{4096}$ & $8.1762 \mathrm{e}-12$ & 4.061 & $3.3763 \mathrm{e}-12$ & 4.090 \\
\hline \hline \multirow{2}{*}{1.8} & \multirow{2}{*}{1.8} & $\frac{1}{8}$ & $\frac{1}{64}$ & $3.0917 \mathrm{e}-08$ & $*$ & $1.5445 \mathrm{e}-08$ & $*$ \\
& & $\frac{1}{16}$ & $\frac{1}{256}$ & $1.9047 \mathrm{e}-09$ & 4.021 & $9.6607 \mathrm{e}-10$ & 3.999 \\
& $\frac{1}{32}$ & $\frac{1}{1024}$ & $1.1627 \mathrm{e}-10$ & 4.034 & $5.8275 \mathrm{e}-11$ & 4.051 \\
& $\frac{1}{64}$ & $\frac{1}{4096}$ & $6.8754 \mathrm{e}-12$ & 4.080 & $3.4108 \mathrm{e}-12$ & 4.095 \\
\hline
\end{tabular}

We use the method (2.17)-(2.19) (abbr. BCIM) to solve Example 4.1 with several values of $h, \tau$ and $\alpha, \beta$, respectively, the numerical results are listed in Table 4.1-Table 4.2. From the results, we can affirm that the fourth order in spatial direction and the second order in temporal direction are in consistent with our theoretical analysis.

For contrast, we also apply the methods (abbr. ADIM and CDIM) in [19] and [21] to solve Example 4.1, respectively. The numerical results are listed in Table 4.3-Table 4.5. Numerical results show that BCIM has the more 
Table 4.2: Errors and corresponding temporal observation orders of BCIM for $\kappa_{1}=2, \kappa_{2}=4$.

\begin{tabular}{cccccccc}
\hline$\alpha$ & $\beta$ & $h$ & $\tau$ & $\|\eta(h, \tau)\|_{\infty}$ & Rate $_{\tau}^{\infty}$ & $\|\eta(h, \tau)\|$ & Rate $_{\tau}$ \\
\hline \multirow{2}{*}{1.1} & \multirow{2}{*}{1.5} & $\frac{1}{200}$ & $\frac{1}{10}$ & $1.8993 \mathrm{e}-07$ & $*$ & $4.9124 \mathrm{e}-08$ & $*$ \\
& & $\frac{1}{200}$ & $\frac{1}{20}$ & $4.5950 \mathrm{e}-08$ & 2.047 & $1.1901 \mathrm{e}-08$ & 2.045 \\
& & $\frac{1}{200}$ & $\frac{1}{40}$ & $1.1396 \mathrm{e}-08$ & 2.012 & $2.9524 \mathrm{e}-09$ & 2.011 \\
& & $\frac{1}{200}$ & $\frac{1}{80}$ & $2.8441 \mathrm{e}-09$ & 2.002 & $7.3692 \mathrm{e}-10$ & 2.002 \\
\hline \hline \multirow{2}{*}{1.3} & \multirow{2}{*}{1.7} & $\frac{1}{200}$ & $\frac{1}{10}$ & $2.6925 \mathrm{e}-07$ & $*$ & $6.9248 \mathrm{e}-08$ & $*$ \\
& & $\frac{1}{200}$ & $\frac{1}{20}$ & $6.3829 \mathrm{e}-08$ & 2.077 & $1.6430 \mathrm{e}-08$ & 2.075 \\
& & $\frac{1}{200}$ & $\frac{1}{40}$ & $1.5759 \mathrm{e}-08$ & 2.018 & $4.0577 \mathrm{e}-09$ & 2.018 \\
& & $\frac{1}{200}$ & $\frac{1}{80}$ & $3.9284 \mathrm{e}-09$ & 2.004 & $1.0115 \mathrm{e}-09$ & 2.004 \\
\hline \hline \multirow{2}{*}{1.5} & \multirow{2}{*}{1.9} & $\frac{1}{200}$ & $\frac{1}{10}$ & $3.9090 \mathrm{e}-07$ & $*$ & $1.0050 \mathrm{e}-07$ & $*$ \\
& & $\frac{1}{200}$ & $\frac{1}{20}$ & $8.9948 \mathrm{e}-08$ & 2.120 & $2.3066 \mathrm{e}-08$ & 2.123 \\
& & $\frac{1}{200}$ & $\frac{1}{40}$ & $2.2049 \mathrm{e}-08$ & 2.028 & $5.6540 \mathrm{e}-09$ & 2.028 \\
& & $\frac{1}{200}$ & $\frac{1}{80}$ & $5.4863 \mathrm{e}-09$ & 2.007 & $1.4069 \mathrm{e}-09$ & 2.007 \\
\hline \hline \multirow{2}{*}{1.8} & \multirow{2}{*}{1.8} & $\frac{1}{200}$ & $\frac{1}{10}$ & $5.3208 \mathrm{e}-07$ & $*$ & $1.3671 \mathrm{e}-07$ & $*$ \\
& & $\frac{1}{200}$ & $\frac{1}{20}$ & $1.2043 \mathrm{e}-07$ & 2.143 & $3.0554 \mathrm{e}-08$ & 2.162 \\
& & $\frac{1}{200}$ & $\frac{1}{40}$ & $2.9191 \mathrm{e}-08$ & 2.045 & $7.3857 \mathrm{e}-09$ & 2.049 \\
& & $\frac{1}{200}$ & $\frac{1}{80}$ & $7.2445 \mathrm{e}-09$ & 2.011 & $1.8319 \mathrm{e}-09$ & 2.011 \\
\hline
\end{tabular}

accurate solutions than ADIM and CDIM with the same conditions. It is obvious to find from Table 4.5 that the three schemes generate the same accuracy for the same temporal grid-size, while the BCIM scheme needs fewer spatial grid points and less CPU time than CDIM and ADIM. This means that BCIM scheme reduces storage requirement and CPU time successfully. All the computations were carried out using MATLAB R2014a software on a HP 288 Pro G2 MT computer, Intel(R) Core(TM) i5-6500, 3.2 GHz CPU machine and 8 GB RAM.

Table 4.3: Errors of numerical methods for $\kappa_{1}=\kappa_{2}=0.5$.

\begin{tabular}{|c|c|c|c|c|c|c|c|}
\hline \multirow{2}{*}{$\alpha$} & \multirow{2}{*}{$\beta$} & \multirow{2}{*}{$h$} & \multirow{2}{*}{$\tau$} & \multicolumn{2}{|c|}{ BCIM } & \multicolumn{2}{|c|}{ ADIM[19] } \\
\hline & & & & $\|\eta(h, \tau)\|_{\infty}$ & $\|\eta(h, \tau)\|$ & $\|\eta(h, \tau)\|_{\infty}$ & $\|\eta(h, \tau)\|$ \\
\hline \multirow[t]{4}{*}{1.1} & 1.1 & $\frac{1}{40}$ & $\frac{1}{40}$ & $2.1747 \mathrm{e}-09$ & $5.7548 \mathrm{e}-10$ & $1.7085 \mathrm{e}-06$ & $4.4962 \mathrm{e}-07$ \\
\hline & & $\frac{1}{80}$ & $\frac{1}{80}$ & $5.4848 \mathrm{e}-10$ & $1.4583 \mathrm{e}-10$ & $1.0019 \mathrm{e}-06$ & $2.5965 \mathrm{e}-07$ \\
\hline & & $\frac{1}{160}$ & $\frac{1}{160}$ & $1.4706 \mathrm{e}-10$ & $3.9499 \mathrm{e}-11$ & $5.5006 \mathrm{e}-07$ & $1.4147 \mathrm{e}-07$ \\
\hline & & $\frac{1}{320}$ & $\frac{1}{320}$ & $4.7129 \mathrm{e}-11$ & $1.3134 \mathrm{e}-11$ & $2.8951 \mathrm{e}-07$ & $7.4208 \mathrm{e}-08$ \\
\hline \multirow[t]{4}{*}{1.5} & 1.5 & $\frac{1}{40}$ & $\frac{1}{40}$ & $3.5315 \mathrm{e}-09$ & $9.0144 \mathrm{e}-10$ & $4.6111 \mathrm{e}-07$ & $1.1639 \mathrm{e}-07$ \\
\hline & & $\frac{1}{80}$ & $\frac{1}{80}$ & $8.8246 \mathrm{e}-10$ & $2.2586 \mathrm{e}-10$ & $2.4185 \mathrm{e}-07$ & $6.0717 \mathrm{e}-08$ \\
\hline & & $\frac{1}{160}$ & $\frac{1}{160}$ & $2.2544 \mathrm{e}-10$ & $5.7922 \mathrm{e}-11$ & $1.2391 \mathrm{e}-07$ & $3.1030 \mathrm{e}-08$ \\
\hline & & $\frac{1}{320}$ & $\frac{1}{320}$ & $6.1618 \mathrm{e}-11$ & $1.6083 \mathrm{e}-11$ & $6.2718 \mathrm{e}-08$ & $1.5688 \mathrm{e}-08$ \\
\hline \multirow[t]{4}{*}{1.9} & 1.9 & $\frac{1}{40}$ & $\frac{1}{40}$ & $6.4489 \mathrm{e}-09$ & $1.6269 \mathrm{e}-09$ & $4.7529 \mathrm{e}-07$ & $1.1933 \mathrm{e}-07$ \\
\hline & & $\frac{1}{80}$ & $\frac{1}{80}$ & $1.6102 \mathrm{e}-09$ & $4.0648 \mathrm{e}-10$ & $2.5926 \mathrm{e}-07$ & $6.5003 \mathrm{e}-08$ \\
\hline & & $\frac{1}{160}$ & $\frac{1}{160}$ & $4.0480 \mathrm{e}-10$ & $1.0224 \mathrm{e}-10$ & $1.3611 \mathrm{e}-07$ & $3.4169 \mathrm{e}-08$ \\
\hline & & $\frac{1}{320}$ & $\frac{1}{320}$ & $1.0376 \mathrm{e}-10$ & $2.6251 \mathrm{e}-11$ & $6.9853 \mathrm{e}-08$ & $1.7559 \mathrm{e}-08$ \\
\hline
\end{tabular}

Example 4.2. Consider the following fractional FitzHugh-Nagumo model[33]

$$
\left\{\begin{array}{l}
\frac{\partial u}{\partial t}=\kappa_{1} \frac{\partial^{\alpha} u}{\partial \mid x^{\alpha}}+\kappa_{2} \frac{\partial^{\beta} u}{\partial \mid y^{\beta}}+u(1-u)(u-\mu)-w, \quad(x, y, t) \in(0,2.5) \times(0,2.5) \times(0, T], \\
\frac{\partial w}{\partial t}=\varepsilon(\lambda u-\gamma w-\delta), \quad(x, y, t) \in(0,2.5) \times(0,2.5) \times(0, T],
\end{array}\right.
$$


Table 4.4: Errors and corresponding observation orders of numerical methods for $\kappa_{1}=\kappa_{2}=1.5$.

\begin{tabular}{|c|c|c|c|c|c|c|c|}
\hline \multirow{2}{*}{$\alpha$} & \multirow{2}{*}{$\beta$} & \multirow{2}{*}{$h$} & \multirow{2}{*}{$\tau$} & \multicolumn{2}{|c|}{ BCIM } & \multicolumn{2}{|c|}{ CDIM[21] } \\
\hline & & & & $\|\eta(h, \tau)\|$ & Rate $_{h}$ & $\|\eta(h, \tau)\|$ & Rate $_{h}$ \\
\hline \multirow[t]{4}{*}{1.1} & \multirow[t]{4}{*}{1.1} & \multirow{4}{*}{$\begin{array}{l}\frac{1}{5} \\
\frac{1}{10} \\
\frac{1}{20} \\
\frac{1}{40} \\
\end{array}$} & $\frac{1}{25}$ & $4.1999 \mathrm{e}-08$ & $*$ & $1.0670 \mathrm{e}-07$ & $*$ \\
\hline & & & $\frac{1}{100}$ & $2.6112 \mathrm{e}-09$ & 4.008 & $2.7498 \mathrm{e}-08$ & 1.956 \\
\hline & & & $\frac{1}{400}$ & $1.5119 \mathrm{e}-10$ & 4.110 & $7.0472 \mathrm{e}-09$ & 1.964 \\
\hline & & & $\frac{1}{1600}$ & $9.1834 \mathrm{e}-12$ & 4.041 & $1.7749 \mathrm{e}-09$ & 1.989 \\
\hline \multirow[t]{4}{*}{1.5} & \multirow[t]{4}{*}{1.5} & \multirow{4}{*}{$\begin{array}{l}\frac{1}{5} \\
\frac{1}{10} \\
\frac{1}{20} \\
\frac{1}{40} \\
\end{array}$} & $\frac{1}{25}$ & $6.6479 \mathrm{e}-08$ & $*$ & $1.8701 \mathrm{e}-07$ & $*$ \\
\hline & & & $\frac{1}{100}$ & $4.3835 \mathrm{e}-09$ & 3.923 & $5.3111 \mathrm{e}-08$ & 1.816 \\
\hline & & & $\frac{1}{400}$ & $2.5617 \mathrm{e}-10$ & 4.097 & $1.4296 \mathrm{e}-08$ & 1.893 \\
\hline & & & $\frac{1}{1600}$ & $1.4680 \mathrm{e}-11$ & 4.125 & $3.6608 \mathrm{e}-09$ & 1.965 \\
\hline \multirow[t]{4}{*}{1.9} & \multirow[t]{4}{*}{1.9} & \multirow{4}{*}{$\begin{array}{l}\frac{1}{5} \\
\frac{1}{10} \\
\frac{1}{20} \\
\frac{1}{40}\end{array}$} & $\frac{1}{25}$ & $9.8521 \mathrm{e}-08$ & * & $3.2851 \mathrm{e}-07$ & $*$ \\
\hline & & & $\frac{1}{100}$ & $7.1405 \mathrm{e}-09$ & 3.786 & $1.0507 \mathrm{e}-07$ & 1.645 \\
\hline & & & $\frac{1}{400}$ & $4.4896 \mathrm{e}-10$ & 3.991 & $3.0700 \mathrm{e}-08$ & 1.775 \\
\hline & & & $\frac{1}{1600}$ & $2.7350 \mathrm{e}-11$ & 4.037 & $8.1275 \mathrm{e}-09$ & 1.917 \\
\hline
\end{tabular}

Table 4.5: Errors and corresponding CPU time costs of numerical methods for $\kappa_{1}=\kappa_{2}=0.5$.

\begin{tabular}{|c|c|c|c|c|c|c|c|c|c|c|c|}
\hline \multirow{2}{*}{$\alpha$} & \multirow{2}{*}{$\beta$} & \multirow[t]{2}{*}{$\tau$} & \multicolumn{3}{|c|}{$\overline{\text { BCIM }}$} & \multicolumn{3}{|c|}{ CDIM[21] } & \multicolumn{3}{|c|}{ ADIM[19] } \\
\hline & & & $h$ & $\|\eta(h, \tau)\|_{\infty}$ & CPU(s) & $h$ & $\|\eta(h, \tau)\|_{\infty}$ & CPU(s) & $h$ & $\|\eta(h, \tau)\|_{\infty}$ & CPU(s) \\
\hline \multirow[t]{6}{*}{1.1} & 1.1 & $\frac{1}{144}$ & $\frac{1}{12}$ & $2.4853 \mathrm{e}-09$ & 1.480 & $\frac{1}{100}$ & $4.4803 e-08$ & 9.506 & $\frac{1}{144}$ & $6.0451 \mathrm{e}-07$ & 16.702 \\
\hline & & $\frac{1}{196}$ & $\frac{1}{14}$ & $1.3567 \mathrm{e}-09$ & 2.372 & $\frac{1}{100}$ & $3.2719 \mathrm{e}-08$ & 12.839 & $\frac{1}{196}$ & $4.5739 \mathrm{e}-07$ & 45.000 \\
\hline & & $\frac{1}{256}$ & $\frac{1}{16}$ & $8.0620 \mathrm{e}-10$ & 3.665 & $\frac{1}{100}$ & $2.4841 \mathrm{e}-08$ & 16.607 & $\frac{1}{256}$ & $3.5716 \mathrm{e}-07$ & 103.324 \\
\hline & & $\frac{1}{400}$ & $\frac{1}{20}$ & $3.4227 \mathrm{e}-10$ & 7.418 & $\frac{1}{100}$ & $1.5540 \mathrm{e}-08$ & 25.941 & $\frac{1}{400}$ & $2.3410 \mathrm{e}-07$ & 477.229 \\
\hline & & $\frac{1}{576}$ & $\frac{1}{24}$ & $1.7382 \mathrm{e}-10$ & 13.295 & $\frac{1}{100}$ & $1.0471 \mathrm{e}-08$ & 40.944 & $\frac{1}{576}$ & $1.6473 \mathrm{e}-07$ & 2089.920 \\
\hline & & $\frac{1}{784}$ & $\frac{1}{28}$ & $1.0093 \mathrm{e}-10$ & 21.833 & $\frac{1}{100}$ & $7.4096 \mathrm{e}-09$ & 48.423 & $\frac{1}{784}$ & $1.2201 \mathrm{e}-07$ & 7282.158 \\
\hline \multirow[t]{6}{*}{1.5} & 1.5 & $\frac{1}{144}$ & $\frac{1}{12}$ & $3.5317 \mathrm{e}-09$ & 1.452 & $\frac{1}{100}$ & $7.9016 \mathrm{e}-08$ & 8.295 & $\frac{1}{144}$ & $1.3730 \mathrm{e}-07$ & 18.782 \\
\hline & & $\frac{1}{196}$ & $\frac{1}{14}$ & $1.9340 \mathrm{e}-09$ & 2.367 & $\frac{1}{100}$ & $5.7999 \mathrm{e}-08$ & 11.372 & $\frac{1}{196}$ & $1.0161 \mathrm{e}-07$ & 47.309 \\
\hline & & $\frac{1}{256}$ & $\frac{1}{16}$ & $1.0980 \mathrm{e}-09$ & 3.686 & $\frac{1}{100}$ & $4.4223 e-08$ & 14.788 & $\frac{1}{256}$ & $7.8158 \mathrm{e}-08$ & 107.309 \\
\hline & & $\frac{1}{400}$ & $\frac{1}{20}$ & $4.4652 \mathrm{e}-10$ & 7.369 & $\frac{1}{100}$ & $2.7885 e-08$ & 23.498 & $\frac{1}{400}$ & $5.0297 \mathrm{e}-08$ & 465.193 \\
\hline & & $\frac{1}{576}$ & $\frac{1}{24}$ & $2.0591 \mathrm{e}-10$ & 13.497 & $\frac{1}{100}$ & $1.8946 \mathrm{e}-08$ & 33.295 & $\frac{1}{576}$ & $3.5032 \mathrm{e}-08$ & 2020.553 \\
\hline & & $\frac{1}{784}$ & $\frac{1}{28}$ & $1.1094 \mathrm{e}-10$ & 22.479 & $\frac{1}{100}$ & $1.3534 \mathrm{e}-08$ & 45.220 & $\frac{1}{784}$ & $2.5783 \mathrm{e}-08$ & 7338.858 \\
\hline \multirow[t]{6}{*}{1.9} & 1.9 & $\frac{1}{144}$ & $\frac{1}{12}$ & $5.7978 \mathrm{e}-09$ & 1.450 & $\frac{1}{100}$ & $1.4748 \mathrm{e}-07$ & 8.290 & $\frac{1}{144}$ & $1.5039 \mathrm{e}-07$ & 19.943 \\
\hline & & $\frac{1}{196}$ & $\frac{1}{14}$ & $3.2021 \mathrm{e}-09$ & 2.364 & $\frac{1}{100}$ & $1.0932 \mathrm{e}-07$ & 11.539 & $\frac{1}{196}$ & $1.1216 \mathrm{e}-07$ & 47.362 \\
\hline & & $\frac{1}{256}$ & $\frac{1}{16}$ & $1.8549 \mathrm{e}-09$ & 3.619 & $\frac{1}{100}$ & $8.4002 \mathrm{e}-08$ & 14.873 & $\frac{1}{256}$ & $8.6739 \mathrm{e}-08$ & 108.434 \\
\hline & & $\frac{1^{6}}{400}$ & $\begin{array}{l}\frac{1}{20} \\
20\end{array}$ & $7.6365 \mathrm{e}-10$ & 7.375 & $\frac{1}{100}$ & $5.3645 \mathrm{e}-08$ & 23.170 & $\frac{2^{2}}{400}$ & $5.6183 \mathrm{e}-08$ & 477.779 \\
\hline & & $\frac{1}{576}$ & $\frac{1}{24}$ & $3.6296 \mathrm{e}-10$ & 13.281 & $\frac{1}{100}$ & $3.6878 \mathrm{e}-08$ & 33.192 & $\frac{1}{576}$ & $3.9275 \mathrm{e}-08$ & 2074.364 \\
\hline & & $\frac{1}{784}$ & $\frac{1}{28}$ & $1.9712 \mathrm{e}-10$ & 21.817 & $\frac{1}{100}$ & $2.6671 \mathrm{e}-08$ & 45.235 & $\frac{1}{784}$ & $2.8971 \mathrm{e}-08$ & 7300.367 \\
\hline
\end{tabular}


where $\delta=0, \mu=0.1, \gamma=1, \varepsilon=0.01, \lambda=0.5$, and $\kappa_{1}, \kappa_{2}$ are nonnegative diffusion coefficients.

The initial-boundary conditions of (4.2a) are taken as

$$
\begin{aligned}
& u(x, y, 0)= \begin{cases}1, & (x, y) \in(0,1.25] \times(0,1.25), \\
0, & \text { elsewhere },\end{cases} \\
& w(x, y, 0)= \begin{cases}0, & (x, y) \in(0,2.5) \times(0,1.25), \\
0.1, & (x, y) \in(0,2.5) \times[1.25,2.5),\end{cases}
\end{aligned}
$$

and

$$
\begin{aligned}
& u(0, y, t)=u(2.5, y, t)=u(x, 0, t)=u(x, 2.5, t)=0, \quad 0 \leq x, y \leq 2.5, \quad 0<t \leq T . \\
& w(0, y, t)=w(2.5, y, t)=w(x, 0, t)=w(x, 2.5, t)=0,
\end{aligned}
$$

Furthermore, BCIM can also be extended to solve the fractional FitzHugh-Nagumo model which is applied for depicting the propagation of the electrical potential in heterogeneous cardiac tissue. In the simulation, we set the parameters $M_{1}=M_{2}=200, N=2000, T=1000$, the results of the simulation at $t=1000$ are shown in Figure 4.1 and Figure 4.2. We observed that the numerical solution of fractional FitzHugh-Nagumo model is related to the fractional orders $\alpha$ and $\beta$. The more details of fractional FitzHugh-Nagumo model can refer to [33].
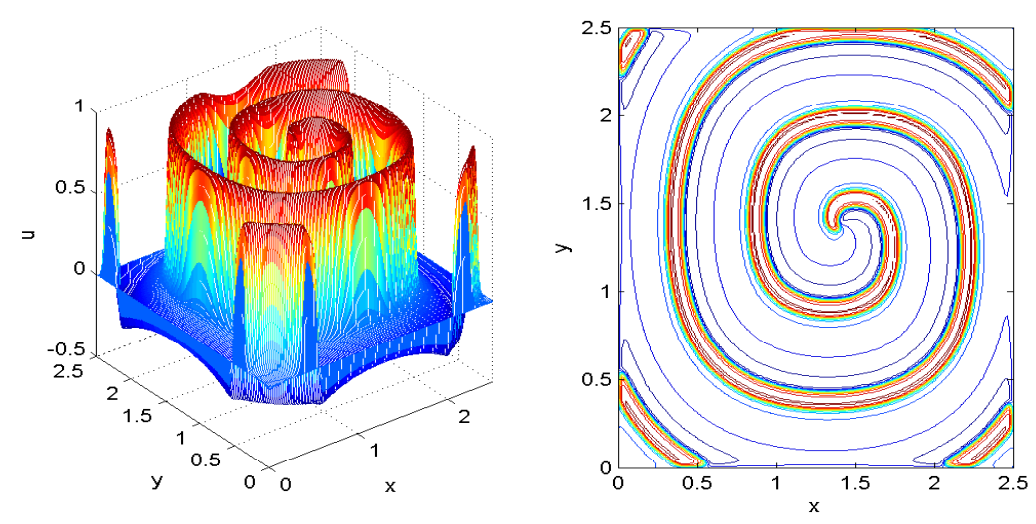

Figure 4.1: Numerical solution of the FitzHugh-Nagumo model with $\kappa_{1}=\kappa_{2}=1 e-4, \alpha=1.7, \beta=1.7$ at $t=1000$.
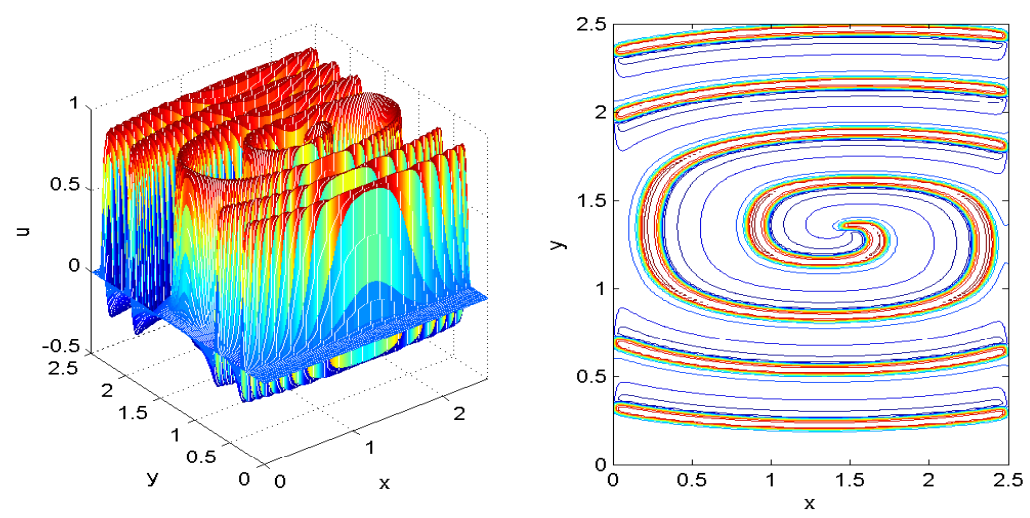

Figure 4.2: Numerical solution of the FitzHugh-Nagumo model with $\kappa_{1}=\kappa_{2}=1 e-4, \alpha=1.8, \beta=1.5$ at $t=1000$. 


\section{Conclusion}

In this paper, a compact ADI finite difference scheme is constructed for the two dimensional Riesz space fractional nonlinear reaction-diffusion equation based on the linearized approximations for nonlinear source term. It's proved that the proposed method is stable and convergent with second-order temporal accuracy and fourth-order spatial accuracy by energy method. Finally, the numerical tests verified the correctness of the theoretical analysis and effectiveness of the proposed scheme by comparing with numerical schemes in $[19,21]$.

\section{Acknowledgements}

The authors would like to express the thanks to the referees for their valuable comments and suggestions.

\section{Disclosure statement}

No potential conflict of interest was reported by the authors.

\section{Funding}

This work is supported by National Science Foundation of China (No. 11671343), and Project of Scientific Research Fund of Hunan Provincial Science and Technology Department (No. 2018WK4006).

\section{References}

\section{References}

[1] X. Zhao, Z. Sun, Z. Hao, A fourth-order compact ADI scheme for 2D nonlinear space fractional Schrödinger equation, SIAM J. Sci. Comput., 36 (2014), pp. 2865-2886

[2] Y. Zhang, H. Ding, High-order algorithm for the two-dimension Riesz space-fractional diffusion equation, Int. J. Comput. Math., 94 (2017), pp. 2063-2073.

[3] H. Liao, P. Lyu, S. Vong, Second-order BDF time approximation for Riesz space-fractional diffusion equations, Int. J. Comput. Math., 95 (2017), pp. 144-158.

[4] H. Chen, S. Gan, D. Xu, Q. Liu, A second-order BDF compact difference scheme for fractional-order Volterra equation, Int. J. Comput. Math., 93 (2015), pp. 1140-1154.

[5] M. Meerschaert, C. Tadjeran, Finite difference approximations for fractional advection-dispersion equations, J. Comput. Appl. Math., 172 (2004), pp. 65-77.

[6] L. Feng, P. Zhuang, F. Liu, I. Turner, J. Li, High-order numerical methods for the Riesz space fractional advection-dispersion equations, Comput. Math. Appl., (2016), http://dx.doi.org/10.1016/j.camwa.2016.01.015. Available online 19 February 2016.

[7] W. Tian, H. Zhou, W. Deng, A class of second order difference approximations for solving space fractional diffusion equations, Math. Comput., 84 (2015), pp. 1703-1727.

[8] P. Wang, C. Huang, An implicit midpoint difference scheme for the fractional Ginzburg-Landau equation, J. Comput. Phys., 312 (2016), pp. 31-49.

[9] H. Ding, C. Li, High-order numerical algorithms for Riesz derivatives via constructing new generating functions, J. Sci. Comput., 71 (2017), pp. 759-784.

[10] A. Kilbas, H. Srivastava, J. Trujillo, Theory and Applications of Fractional Differential Equations, Elsevier Science Limited, 2006.

[11] C. Li, Y. Wu, R. Ye, Recent advances in applied nonlinear dynamics with numerical analysis: fractional dynamics, network dynamics, classical dynamics and fractal dynamics with their numerical simulations, World Scientific, 2013.

[12] C. Li, F. Zeng, Numerical methods for fractional calculus, Chapman and Hall/CRC, Boca Raton, 2015.

[13] C. Li, A. Chen, Numerical methods for fractional partial differential equations, Int. J. Comput. Math., 95 (2018), pp. 1048-1099.

[14] M. Ortigueira, Riesz potential operators and inverses via fractional centred derivatives, Int. J. Math. Math. Sci., 2006 (2006) 12 (Article ID 48391).

[15] C. Çelik, M. Duman, Crank-Nicolson method for the fractional diffusion equation with the Riesz fractional derivative, J. Comput. Phys., 231 (2012), pp. 1743-1750.

[16] S. Arshad, W. Bu, J. Huang, Y. Tang, Y. Zhao, Finite difference method for time-space linear and nonlinear fractional diffusion equations, Int. J. Comput. Math., 95 (2018), pp. 202-217.

[17] H. Zhou, W. Tian, W. Deng, Quasi-compact finite difference schemes for space fractional diffusion equations, J. Sci. Comput., 56 (2013), pp. 45-66.

[18] Z. Hao, Z. Sun, W. Cao, A fourth-order approximation of fractional derivatives with its applications, J. Comput. Phys., 281 (2015), pp. $787-805$. 
[19] F. Liu, S. Chen, I. Turner, K. Burrage, V. Anh, Numerical simulation for two-dimensional riesz space fractional diffusion equations with a nonlinear reaction term, Cent. Eur. J. Phys., 11 (2013), pp. 1221-1232.

[20] F. Zeng, F. Liu, C. Li, K. Burrage, I. Turner, V. Anh, A Crank-Nicolson ADI spectral method for a two-dimensional Riesz space fractional nonlinear reaction-diffusion equation, SIAM J. Numer. Anal., 52 (2014), pp. 2599-2622.

[21] F. Liu, P. Zhuang, I. Turner, V. Anh, K. Burrage, A semi-alternating direction method for a 2-D fractional FitzHugh-Nagumo monodomain model on an approximate irregular domain, J. Comput. Phys., 293 (2015), pp. 252-263.

[22] W. Bu, Y. Tang, Y. Wu, J. Yang, Crank-Nicolson ADI Galerkin finite element method for two-dimensional fractional FitzHugh-Nagumo monodomain model, Appl. Math. Comput., 257 (2015), pp. 355-364.

[23] O. Iyiola, E. Asante-Asamani, K. Furati, A. Khaliq, B. Wade, Efficient time discretization scheme for nonlinear space fractional reactiondiffusion equations, Int. J. Comput. Math., 95 (2018), pp. 1274-1291.

[24] K. Furati, M. Yousuf, A. Khaliq, Fourth-order methods for space fractional reaction-diffusion equations with non-smooth data, Int. J. Comput. Math., 95 (2018), pp. 1240-1256.

[25] M. Yousuf, A second-order efficient L-stable numerical method for space fractional reaction-diffusion equations, Int. J. Comput. Math., 95 (2018), pp. 1408-1422.

[26] X. Cao, X. Cao, L. Wen, The implicit midpoint method for the modified anomalous sub-diffusion equation with a nonlinear source term, J. Comput. Appl. Math., 318 (2017), pp. 199-210.

[27] Y. Li, D. Wang, Improved efficient difference method for the modified anomalous sub-diffusion equation with a nonlinear source term, Int. J. Comput. Math., 94 (2017), pp. 821-840.

[28] Y. Choi, S. Chung, Finite element solutions for the space-fractional diffusion equation with a nonlinear source term, Abstr. Appl. Anal., 2012 (2012), pp. 183-201.

[29] K. Burrage, N. Hale, D. Kay, An efficient implicit FEM scheme for fractional-in-space reaction-diffusion equations, SIAM J. Sci. Comput., 34 (2012), pp. 2145-2172.

[30] S. Chen, F. Liu, X. Jiang, I. Turner, V. Anh, A fast semi-implicit difference method for a nonlinear two-sided space-fractional diffusion equation with variable diffusivity coefficients, Appl. Math. Comput., 257 (2015), pp. 591-601.

[31] H. Choi, S. Chung, Y. Lee, Numerical solutions for space-fractional dispersion equations with nonlinear source terms, Bull. Korean Math. Soc., 47 (2010), pp. 1225-1234.

[32] D. Wang, A. Xiao, W. Yang, Crank-Nicolson difference scheme for the coupled nonlinear Schrödinger equations with the Riesz space fractional derivative, J. Comput. Phys., 242 (2013), pp. 670-681.

[33] A. Bueno-Orovio, D. Kay, K. Burrage, Fourier spectral methods for fractional-in-space reaction-diffusion equations, BIT. Numer. Math., 54 (2014), pp. 937-954.

[34] F. Lin, H. Qu, A Runge-Kutta Gegenbauer spectral method for nonlinear fractional differential equations with Riesz fractional derivatives, Int. J. Comput. Math., 96 (2019), pp. 417-435.

[35] W. Hundsdorfe, Partially implicit BDF2 blends for convection dominated flows, SIAM J. Numer. Anal., 38 (2001), pp. $1763-1783$. 